\title{
Search for high-mass resonances in final states with a lepton and missing transverse momentum at $\sqrt{s}=13 \mathrm{TeV}$
}

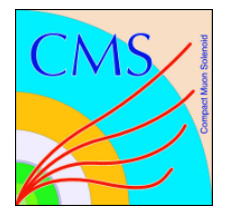

\section{The CMS collaboration}

E-mail: cms-publication-committee-chair@cern.ch

ABSTRACT: A search for new high-mass resonances in proton-proton collisions having final states with an electron or muon and missing transverse momentum is presented. The analysis uses proton-proton collision data collected in 2016 with the CMS detector at the LHC at a center-of-mass energy of $13 \mathrm{TeV}$, corresponding to an integrated luminosity of $35.9 \mathrm{fb}^{-1}$. The transverse mass distribution of the charged lepton-neutrino system is used as the discriminating variable. No significant deviation from the standard model prediction is found. The best limit, from the combination of electron and muon channels, is $5.2 \mathrm{TeV}$ at $95 \%$ confidence level for the mass of a $\mathrm{W}^{\prime}$ boson with the same couplings as those of the standard model $\mathrm{W}$ boson. Exclusion limits of $2.9 \mathrm{TeV}$ are set on the inverse radius of the extra dimension in the framework of split universal extra dimensions. In addition, model-independent limits are set on the production cross section and coupling strength of $\mathrm{W}^{\prime}$ bosons decaying into this final state. An interpretation is also made in the context of an $\mathrm{R}$ parity violating supersymmetric model with a slepton as a mediator and flavor violating decay.

KeYwords: Beyond Standard Model, Hadron-Hadron scattering (experiments)

ArXiv EPrint: 1803.11133 


\section{Contents}

1 Introduction 1

2 The CMS detector 2

3 Physics models and event simulation 3

3.1 Sequential standard model $\mathrm{W}^{\prime}$ boson 4

3.2 Varying coupling strength 4

3.3 Split-UED model 5

3.4 RPV SUSY with scalar lepton mediator 5

4 Event reconstruction $\quad 6$

$\begin{array}{llr}5 & \text { Event selection } & 7\end{array}$

6 Background $\quad 9$

7 Systematic uncertainties 12

8 Interpretation of the results 13

8.1 Statistical analysis 13

8.2 Model-independent cross section limit 13

$\begin{array}{lll}\text { 8.3 Limits on an SSM W' boson } & 15\end{array}$

$\begin{array}{ll}\text { 8.4 Limits on the coupling strength } & 16\end{array}$

$\begin{array}{lll}\text { 8.5 Interpretation in the split-UED model } & 16\end{array}$

$\begin{array}{lll}8.6 & \text { Limits on RPV SUSY } & 17\end{array}$

9 Summary 18

The CMS collaboration $\quad 24$

\section{Introduction}

The standard model (SM) of particle physics describes the properties of all known elementary particles and the forces between them. Five decades of experimental studies have verified its predictions to very high precision. Despite the great success of the SM, theories beyond the SM (BSM) have been invoked to address a variety of open issues. Many SM extensions predict additional heavy gauge bosons, including models with extended gauge sectors [1], and theories with extra spatial dimensions [2,3]. 
The search presented in this paper is sensitive to deviations from the SM prediction in the transverse mass spectrum of events with a charged lepton (electron or muon) and a neutrino. Interpretations of the observations are made in the context of several theoretical models: the production and decay of a $\mathrm{W}^{\prime}$ boson in the sequential standard model (SSM) [4], the production and flavor violating decay of a slepton in an R-parity violating supersymmetry (RPV SUSY) model [5, 6], and the production and decay of a Kaluza-Klein (KK) excitation of the $\mathrm{W}$ boson in a model with split universal extra dimensions (split-UED) [7].

The shape of the distribution is studied using a binned likelihood method. This approach is especially powerful as the examined theories predict different signal event distributions. Although the details differ, all models predict that the signal of a high-mass resonance is present at large transverse masses where SM backgrounds are very small.

The present analysis uses data corresponding to an integrated luminosity of $35.9 \mathrm{fb}^{-1}$ of proton-proton collisions at a center-of-mass energy of $13 \mathrm{TeV}$, recorded in 2016 with the CMS detector at the CERN LHC. The analysis improves upon the sensitivity of its predecessors $[8,9]$, benefiting from the increased energy and luminosity of the LHC. Previous searches $[10,11]$ have not found evidence for deviations from the SM prediction for the transverse mass distribution.

\section{The CMS detector}

The central feature of the CMS apparatus is a superconducting solenoid of $6 \mathrm{~m}$ internal diameter, providing a magnetic field of $3.8 \mathrm{~T}$. Within the solenoid volume are a silicon pixel and strip tracker, a lead tungstate crystal electromagnetic calorimeter (ECAL), and a brass and scintillator hadron calorimeter (HCAL), each composed of a barrel and two endcap sections. Extensive forward calorimetry complements the coverage provided by the barrel and endcap detectors.

The silicon tracker measures charged particles within the pseudorapidity range $|\eta|<$ 2.5. It consists of silicon pixel and silicon strip detector modules. The electromagnetic calorimeter consists of 75848 lead tungstate crystals that provide coverage in pseudorapidity $|\eta|<1.48$ in a barrel region and $1.48<|\eta|<3.00$ in two endcap regions. The ECAL energy resolution for electrons with a transverse momentum $p_{\mathrm{T}} \approx 45 \mathrm{GeV}$ from $\mathrm{Z} \rightarrow$ ee decays is better than $2 \%$ in the central region of the ECAL barrel $(|\eta|<0.8)$, and is between $2 \%$ and $5 \%$ elsewhere [12]. For high energies, which are relevant for this analysis, the electron energy resolution slightly improves [13].

Muons are measured in gas-ionization detectors embedded in the steel flux-return yoke outside the solenoid, in the pseudorapidity range $|\eta|<2.4$. Detection is provided using three technologies: drift tube (DT), cathode strip chamber (CSC), and resistive plate chamber (RPC). While the barrel region of $|\eta| \leq 1.1$ is instrumented with DT and RPC, the forward endcaps $(1.1<|\eta|<2.4)$ are equipped with CSC and RPC. A muon from the interaction point will cross four layers of muon chambers, interleaved with steel forming the return yoke of the magnetic field. Every chamber provides reconstructed hits on several detection planes, which are then combined into local track segments, forming the basis of muon reconstruction inside the muon system. Matching muons track segments to tracks 
measured in the silicon tracker results in a relative transverse momentum $\left(p_{\mathrm{T}}\right)$ resolution in the barrel of about $1-2 \%$ for muons with $p_{\mathrm{T}} \lesssim 200 \mathrm{GeV}$ and better than $10 \%$ for high momentum muons of $p_{\mathrm{T}} \sim 1 \mathrm{TeV}$ [14].

Jets are reconstructed offline from the energy deposits in the calorimeter towers, clustered using the anti- $k_{\mathrm{T}}$ algorithm $[15,16]$ with a distance parameter of 0.4 . In this process, the contribution from each calorimeter tower is assigned a momentum, the absolute value and the direction of which are given by the energy measured in the tower, and the coordinates of the tower. The raw jet energy is obtained from the sum of the tower energies, and the raw jet momentum by the vectorial sum of the tower momenta, which results in a nonzero jet mass. The raw jet energies are then corrected to establish a relative uniform response of the calorimeter in $\eta$ and a calibrated absolute response in transverse momentum $p_{\mathrm{T}}$.

The CMS experiment has a two-level trigger system [17]. The level-1 trigger, composed of custom hardware processors, selects events of interest using information from the calorimeters and muon detectors and reduces the readout rate from the $40 \mathrm{MHz}$ bunchcrossing frequency to a maximum of $100 \mathrm{kHz}$. The software based high-level trigger uses the full event information, including that from the inner tracker, to reduce the event rate to the $1 \mathrm{kHz}$ that is recorded.

A more detailed description of the CMS detector can be found in ref. [18].

\section{Physics models and event simulation}

Many BSM scenarios predict the existence of new particles that decay with the experimental signature of a charged lepton, $\ell$, and missing transverse momentum, $p_{\mathrm{T}}^{\text {miss }}$, where the latter may flag the presence of a non-interacting particle. The missing transverse momentum, $\vec{p}_{\mathrm{T}}^{\text {miss }}$, is defined as $-\sum \vec{p}_{\mathrm{T}}$ of all reconstructed particles, with $p_{\mathrm{T}}^{\text {miss }}$ being the modulus of $\vec{p}_{\mathrm{T}}^{\text {miss }}$.

The analysis is performed in two channels: $\mathrm{e}+p_{\mathrm{T}}^{\text {miss }}$ and $\mu+p_{\mathrm{T}}^{\text {miss }}$. New particles may be detected as an excess of events in the observed spectrum of transverse mass, defined as

$$
M_{\mathrm{T}}=\sqrt{2 p_{\mathrm{T}}^{\ell} p_{\mathrm{T}}^{\text {miss }}\left(1-\cos \left[\Delta \phi\left(\ell, \vec{p}_{\mathrm{T}}^{\text {miss }}\right)\right]\right)},
$$

where $\Delta \phi\left(\ell, \vec{p}_{\mathrm{T}}^{\text {miss }}\right)$ is the azimuthal opening angle (in radians) between the directions of the missing transverse momentum and the charged lepton. Several new-physics models predict the production of high- $p_{\mathrm{T}}$ leptons, which should be identifiable as an excess in the region of high $M_{\mathrm{T}}$ values where little SM background is expected.

This section summarizes the new physics models used for interpretation of the observations, along with model-specific assumptions and details of the generator programs used for production of simulated signal event samples. All generated events are processed through a full simulation of the CMS detector based on GEANT4 [19], a trigger emulation, and the event reconstruction chain.

All simulated event samples are normalized to the integrated luminosity of the recorded data. The simulation of pileup is included in all event samples by superimposing simulated 

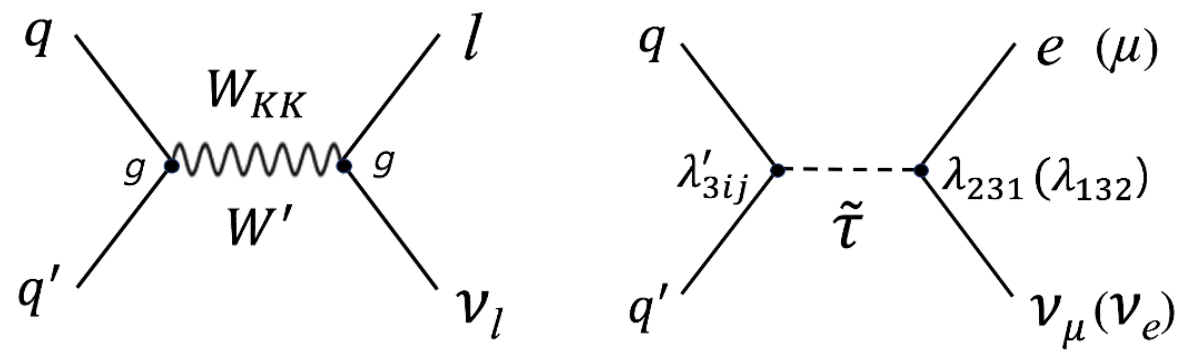

Figure 1. Production and decay of a new heavy boson, an SSM $W^{\prime}$ or a $\mathrm{W}_{\mathrm{KK}}$ (left). The coupling strength, $g$, may vary. In RPV SUSY, a tau slepton $(\widetilde{\tau})$ could also act as a mediator (right) with corresponding $\lambda$ couplings for the decay that are different for the two final states, denoted by $\lambda_{231}$ and $\lambda_{132}$ for the electron and muon final states, respectively.

minimum bias interactions onto all simulated events. For the data set used, the average number of interactions per bunch-crossing after selection is about 20, with a maximum of 55 .

\subsection{Sequential standard model $\mathrm{W}^{\prime}$ boson}

The SSM [4] has been used as a benchmark model for experimental $\mathrm{W}^{\prime}$ boson searches for more than two decades. The Feynman diagram for the production and decay of a $\mathrm{W}^{\prime}$ boson is depicted in figure 1 (left). In accordance with previous analyses, no interference with the SM W boson is considered.

In the SSM, the $\mathrm{W}^{\prime}$ boson is considered to be a heavy analogue of the SM W boson, with similar decay modes and branching fractions. These are modified by the presence of the $t \bar{b}$ decay channel, which opens up for $\mathrm{W}^{\prime}$ boson masses above $180 \mathrm{GeV}$. Dedicated searches in the $t \bar{b}$ channel are described in refs. [20-22]. For this search, the given assumptions yield a predicted branching fraction $(\mathcal{B})$ of about $8.5 \%$ for each of the leptonic channels studied. The width of a $1 \mathrm{TeV} \mathrm{W}^{\prime}$ boson would be about $33 \mathrm{GeV}$. Decays of the $\mathrm{W}^{\prime}$ boson via $\mathrm{WZ}$ are assumed to be suppressed. Dedicated searches for these decays can be found in refs. [23, 24].

The signature of a $\mathrm{W}^{\prime}$ boson is a Jacobian peak in the transverse mass distribution, similar to that of the SM W boson, but at a higher mass. Because of constraints from the parton distribution functions (PDF) the phase space for production of very massive $\mathrm{W}^{\prime}$ bosons in pp collisions at $13 \mathrm{TeV}$ is reduced, leading to a large fraction of such $\mathrm{W}^{\prime}$ bosons being produced off-shell, at lower masses.

The simulation of data samples in the SSM is performed at leading order (LO) with PYTHIA 8.212 [25], using the NNPDF2.3 PDF set [26, 27] and tune CUETP8M1 [28]. The simulated masses range from $400 \mathrm{GeV}$ to $6 \mathrm{TeV}$, where the lower mass matches the beginning of the sensitive region as determined by the trigger thresholds. A $\mathrm{W}^{\prime}$ boson mass-dependent $\mathrm{K}$-factor is used to correct for next-to-next-to-leading order (NNLO) QCD multijet cross sections, calculated using FEWZ $3.1[29,30]$. The K-factor varies from 1.363 to 1.140.

\subsection{Varying coupling strength}

The $\mathrm{W}^{\prime}$ boson coupling strength, $g_{\mathrm{W}^{\prime}}$, is usually given in terms of the SM weak coupling strength $g_{\mathrm{W}}$. If the $\mathrm{W}^{\prime}$ is a copy of the SM W boson, their coupling ratio is $g_{\mathrm{W}^{\prime}} / g_{\mathrm{W}}=1$ 
and the SSM $\mathrm{W}^{\prime}$ theoretical cross sections, signal shapes, and widths apply. However, different couplings are possible. Because of the dependence of the width of a particle on its coupling, and the consequent effect on the $M_{\mathrm{T}}$ distribution, a limit can also be set on the coupling strength. For this study, signal samples for a range of coupling ratios, $g_{\mathrm{W}^{\prime}} / g_{\mathrm{W}}=10^{-2}$ to 3 , are simulated in LO with MADGRAPH 5 (v1.5.11) [31]. These signals exhibit different widths as well as different cross sections. The generated distributions of the PYTHIA samples are reweighted to take into account the decay width dependence, thus providing the appropriate reconstructed $M_{\mathrm{T}}$ distributions. For $g_{\mathrm{W}^{\prime}} / g_{\mathrm{W}}=1$ the theoretical LO cross sections apply and this coupling strength was used to compare the standard SSM samples with the reweighted ones, allowing the reweighting method to be verified. For $g_{\mathrm{W}^{\prime}} / g_{\mathrm{W}} \neq 1$ the theoretical cross sections scale with the coupling strength squared.

\subsection{Split-UED model}

The leptonic final states under study may also be interpreted in the framework of universal extra dimensions with fermions propagating in the bulk, known as split-UED model [7, 32]. This is a model based on an extended space-time with an additional compact fourth spatial dimension of radius $R$, and a bulk mass parameter of the fermion field in five dimensions, $\mu$. In this model all SM particles have corresponding Kaluza-Klein (KK) partners, for instance $\mathrm{W}_{\mathrm{KK}}^{(\mathrm{n})}$, where the superscript denotes the $\mathrm{n}^{\text {th }} \mathrm{KK}$ excitation mode. Only KK-even KK modes of $\mathrm{W}_{\mathrm{KK}}^{(\mathrm{n})}$ couple to SM fermions, owing to KK-parity conservation.

In the split-UED model the parameter $\mu$ is assumed to be non-zero, following refs. [7, 32]. The mass of the $\mathrm{W}_{\mathrm{KK}}^{(\mathrm{n})}$ is determined by $\mathrm{M}\left(\mathrm{W}_{\mathrm{KK}}^{(\mathrm{n})}\right)=\sqrt{M_{\mathrm{W}}+(n / R)}$, i.e., a larger radius $(R)$ corresponds to smaller KK masses. The mass of KK fermions depends on the bulk mass parameter $\mu$. The product of the cross section of the $\mathrm{W}_{\mathrm{KK}}^{(\mathrm{n})}$ production and the branching fraction to standard model fermions goes to zero as $\mu$ goes to zero.

For the mode $n=2$, the decay of $\mathrm{W}_{\mathrm{KK}}^{(2)}$ to leptons is kinematically identical to the $\mathrm{SSM} \mathrm{W}^{\prime}$ boson decay, and the observed limits obtained from the $\mathrm{W}^{\prime} \rightarrow \mathrm{e} \nu$ and $\mathrm{W}^{\prime} \rightarrow \mu \nu$ searches can be reinterpreted directly in terms of the $\mathrm{W}_{\mathrm{KK}}^{(2)}$ boson mass, taking into account the difference in widths in the simulation. The Feynman diagram in figure 1 (left) shows this process. The simulation is performed at leading order with PYTHIA 8.212. The massdependent $\mathrm{K}$-factors from the $\mathrm{SSM} \mathrm{W}^{\prime}$ interpretation are used. This is possible since the signal shapes of a $\mathrm{W}_{\mathrm{KK}}^{(2)}$ and a SSM $\mathrm{W}^{\prime}$ correspond to each other. The signal samples are generated with the parameters $1 / R=200$ to $3000 \mathrm{GeV}$ and $\mu=50$ to $10000 \mathrm{GeV}$. The $1 / R$ range corresponds to the mass of $\mathrm{W}_{\mathrm{KK}}^{(2)}$ from approximately 400 to $6000 \mathrm{GeV}$.

\subsection{RPV SUSY with scalar lepton mediator}

This model assumes a SUSY scalar lepton $(\widetilde{\ell})$ as a mediator, with subsequent R-parity and lepton flavor violating decay to a charged lepton and a neutrino $[5,6]$. The analysis studies the cases where a tau slepton decays to e $+\nu_{\mu}$ or to $\mu+\nu_{\mathrm{e}}$. The Feynman diagram is depicted on the right of figure 1 for the two decay channels under study. While on the production side, the coupling is always a version of the hadronic-leptonic RPV coupling $\lambda_{3 i j}^{\prime}$ (which is the coupling to the third generation, in this case the tau slepton), the decay is governed by the leptonic RPV coupling $\lambda_{231}$ for the decay to e $+\nu_{\mu}$, and by $\lambda_{132}$ for the 
decay to $\mu+\nu_{e}$. The values of the couplings may be identical. Signal samples for a range of tau slepton masses, $\mathrm{M}(\widetilde{\tau})$, are simulated with MADGrAPH 5 (v1.5.14) at LO and no higher order effects are considered. Signals are simulated with the parameters $\lambda_{231}, \lambda_{132}$ and $\lambda_{3 i j}^{\prime}=0.05$ to 0.5 for $M(\widetilde{\tau})=400$ to $6000 \mathrm{GeV}$.

\section{Event reconstruction}

The models described in the previous section provide an event signature of a single high- $p_{\mathrm{T}}$ lepton (electron or muon) and a particle that cannot be detected directly, giving rise to the experimentally observed $p_{\mathrm{T}}^{\text {miss }}$. This quantity is measured using a particle-flow (PF) technique [33], that combines measurements from all components of the CMS detector in order to produce particle candidates. The modulus of the vector $p_{\mathrm{T}}$ sum of these candidates defines $p_{\mathrm{T}}^{\text {miss }}$, which is corrected for the jet energy calibration [34, 35]. At high mass, the $p_{\mathrm{T}}^{\text {miss }}$ is mainly determined by the high- $p_{\mathrm{T}}$ lepton in the event.

Electrons are reconstructed as ECAL clusters that are matched to a central track and their identification has been optimized for high- $p_{\mathrm{T}}$ values [36]. Electron candidates are required to be isolated, have an electron-like shape, and be within the acceptance region of the barrel $(|\eta|<1.44)$ or the endcaps $(1.56<|\eta|<2.50)$. This acceptance region avoids the transition region between barrel and endcap parts of the ECAL. Electron isolation in the tracker is ensured by requiring the sum of $p_{\mathrm{T}}$ to be less than $5 \mathrm{GeV}$ for all tracks that are in close proximity to the track of the electron candidate and to originate from the same primary vertex. Only tracks that are within a cone of $\Delta R=\sqrt{(\Delta \phi)^{2}+(\Delta \eta)^{2}}<0.3$ around the electron candidate's track are considered. The reconstructed vertex with the largest value of summed physics-object $p_{\mathrm{T}}^{2}$ is taken to be the primary pp interaction vertex. The physics objects are the jets, clustered using the jet finding algorithm $[15,16]$ with the tracks assigned to the vertex as inputs, and the associated missing transverse momentum, taken as the negative vector sum of the $p_{\mathrm{T}}$ of those jets. As in the tracker isolation calculation, in the calorimeters the sum of energy deposits within a cone $\Delta R<0.3$ around the electron candidate's direction is used as a measure of isolation. It is corrected for the mean energy contribution from additional pp collisions occurring within the same bunch crossing (pileup) [37]. To obtain sufficiently isolated electrons, this calorimeter isolation is required to be below a threshold of $3 \%$ of the electron's transverse momentum. Additionally, the energy deposits in the hadron calorimeter within a cone of $\Delta R=0.15$ around the electron's direction must be less than $5 \%$ of the electron's energy deposit in the ECAL. In order to differentiate between electrons and photons, properties of the track matched to the calorimeter measurement must be consistent with those of an electron originating from the primary vertex. Specifically, there must be $\leq 1$ hit missing in the innermost tracker layers, and the transverse distance to the primary vertex must be $<0.02 \mathrm{~cm}$ (barrel) or $<0.05 \mathrm{~cm}$ (endcap).

The muon system covers the pseudorapidity region $|\eta|<2.4$. The reconstruction of muons is optimized for high- $p_{\mathrm{T}}$ values [36]. Information from the inner tracker and the outer muon system are used together. Each muon track is required to have at least one hit in the pixel detector, at least six tracker layer hits, and segments with hits in two or more muon detector stations. Since segments are typically in consecutive layers separated 
by thick layers of steel, the latter requirement significantly reduces the amount of hadronic punch-through [38]. To reduce background from cosmic ray muons, each muon is required to have a transverse impact parameter of less than $0.02 \mathrm{~cm}$ and a longitudinal distance parameter of less than $0.5 \mathrm{~cm}$ with respect to the primary vertex. In order to suppress muons with mismeasured $p_{\mathrm{T}}$, an additional requirement $\sigma_{p_{\mathrm{T}}} / p_{\mathrm{T}}<0.3$ is applied, where $\sigma_{p_{\mathrm{T}}}$ is the $p_{\mathrm{T}}$ uncertainty from the muon track reconstruction. Muon isolation requires that the scalar $p_{\mathrm{T}}$ sum of all tracks originating from the interaction vertex within a $\Delta R<0.3$ cone around its direction, excluding the muon itself, is less than $10 \%$ of the muon's $p_{\mathrm{T}}$.

In order to determine any differences in the selection efficiencies between observed and simulated data, the efficiencies for both channels are measured using the "tag-and-probe" method [39], with samples of dilepton events from high- $p_{\mathrm{T}} \mathrm{Z}$ boson decays and high-mass Drell-Yan pairs. The overall efficiency in each case includes contributions from the trigger and the lepton reconstruction and identification criteria. The ratio of the efficiencies for data and simulation, denoted as the scale factor $(\mathrm{SF})$, is determined for each channel separately. The SFs that match the electron identification and reconstruction efficiencies in data and simulation are $0.972 \pm 0.006$ (barrel) and $0.983 \pm 0.007$ (endcap). The muon scale factors are also sensitive to differences between simulated samples and data, caused by radiative processes associated with muon interactions in the material of the detector. The corresponding SFs are applied as a function of $\eta$, with uncertainties covering possible lower efficiencies in data and dependent on the muon momentum that, for a muon momentum of $5 \mathrm{TeV}$, range from $3 \%$ for $|\eta|<1.6$ and up to $20 \%$ for $1.6<|\eta|<2.4$.

\section{Event selection}

The event selection follows the approach used in previous CMS analyses [8, 9]. Events are triggered with a single-electron (muon) trigger with trigger thresholds of lepton $p_{\mathrm{T}}>$ $115(50) \mathrm{GeV}$. In addition, high- $p_{\mathrm{T}}$ electrons may be triggered by a single-photon trigger with a threshold of $175 \mathrm{GeV}$ for the photon $p_{\mathrm{T}}$, which is used in a logical "OR" with the single-electron trigger. For the electron channel, the trigger SF is determined to be $0.989 \pm 0.003$ (barrel) and $0.996 \pm 0.003$ (endcap), respectively, for the combination of the single-electron and single-photon triggers. The muon trigger SF accounts for observed differences between the efficiencies in simulation and in data for particular regions of the detector, such as the transition region between the barrel and endcap muon detectors. The SF values, applied as a function of $\eta$, range from 0.92 to unity.

The minimum offline lepton $p_{\mathrm{T}}$ must be above the lepton trigger threshold. For offline reconstruction, electrons are required to have $p_{\mathrm{T}}>130 \mathrm{GeV}$ and to have $|\eta|<1.44$ or $1.56<|\eta|<2.50$. Muons must have $p_{\mathrm{T}}>53 \mathrm{GeV}$ and $|\eta|<2.4$. Signal events are identified by the presence of an isolated high- $p_{\mathrm{T}}$ lepton. Events with a second lepton with $p_{\mathrm{T}}>25 \mathrm{GeV}$ are rejected. In the electron channel, $p_{\mathrm{T}}^{\text {miss }}$ is required to be above $150 \mathrm{GeV}$ to avoid the mismodeled low- $p_{\mathrm{T}}^{\mathrm{miss}}$ region.

In the muon channel, the contribution from t $\bar{t}$ events is further reduced by rejecting events containing six or more jets or events with the leading jet having $p_{\mathrm{T}}>25 \mathrm{GeV}$ 

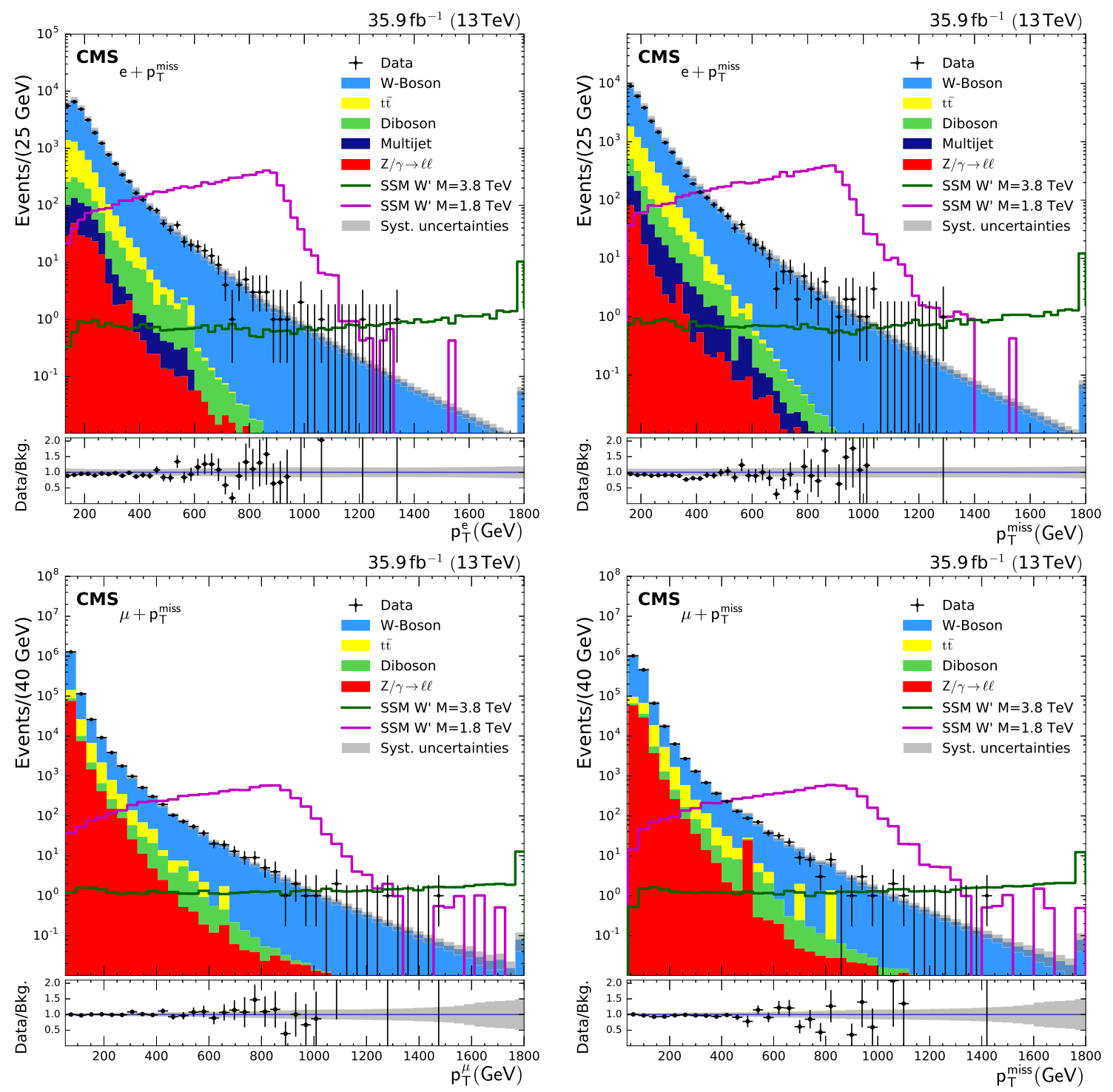

Figure 2. Distributions in $p_{\mathrm{T}}$ (left) and $p_{\mathrm{T}}^{\text {miss }}$ (right), for the electron (upper row) and muon (lower row) for data and for expected SM backgrounds, after applying complete selection criteria. The QCD multijet background in the electron channel is derived from data. The background labelled as "diboson" includes WW, ZZ, and WZ contributions. Also shown are SSM W' signal examples for the two indicated masses. The last bin shows the total overflow. The lower panel shows the ratio of data to prediction and the shaded band includes the systematic uncertainties. 
and $|\eta|<2.5$, consistent with originating from a bottom quark, using the standard CMS b-tagging tools [40].

In the considered models, the lepton and $\vec{p}_{\mathrm{T}}^{\text {miss }}$ are expected to be nearly back-toback in the transverse plane, and balanced in transverse momentum. To incorporate these characteristics in the analysis, additional kinematic criteria select events based on the ratio of the lepton $p_{\mathrm{T}}$ to $p_{\mathrm{T}}^{\text {miss }}$, requiring $0.4<p_{\mathrm{T}} / p_{\mathrm{T}}^{\text {miss }}<1.5$, and on the angular difference between the lepton and $p_{\mathrm{T}}^{\text {miss }}$, with $\Delta \phi\left(\ell, \vec{p}_{\mathrm{T}}^{\text {miss }}\right)>2.5 \approx 0.8 \pi$. The distributions of the lepton $p_{\mathrm{T}}$ and $p_{\mathrm{T}}^{\text {miss }}$ are depicted in figure 2 separately for the electron (upper panels) and muon (lower panels) channels.

For simulated events passing all the selection criteria, the signal efficiency for an SSM $\mathrm{W}^{\prime}$ with no requirement on the reconstructed $M_{\mathrm{T}}$ in the event, is maximal at a value of 0.75 (for both decay channels) for a $\mathrm{W}^{\prime}$ boson mass range $1.5-2.5 \mathrm{TeV}$, and decreases gradually to $\approx 0.60$ for larger and smaller masses. For larger masses, the increasing off-shell production displaces events to lower $M_{\mathrm{T}}$, where the background is larger.

The resulting $M_{\mathrm{T}}$ distributions for the analyzed data sets are shown in figure 3 for the electron and muon channels. The minimum value of $M_{\mathrm{T}}$ is determined by the trigger thresholds, resulting in a choice of $\sim 250$ and $\sim 100 \mathrm{GeV}$ in the electron and muon channels, respectively. Included in figure 3 are the predicted $M_{\mathrm{T}}$ distributions for the accepted SM events, separated into contributions from each background process, along with example signal distributions for the SSM W', split-UED, and SUSY models. For the muon channel, a variable binning commensurate with the energy-dependent $M_{\mathrm{T}}$-resolution is used. The expected systematic uncertainties in the predicted $M_{\mathrm{T}}$ distributions are also shown. The numbers of signal and background events for a selected set of $M_{\mathrm{T}}$ thresholds are shown in table 1 .

No significant excess over the SM expectation is observed in the $M_{\mathrm{T}}$ spectrum. The highest transverse mass events observed have $M_{\mathrm{T}} \sim 2.6$ and $M_{\mathrm{T}} \sim 2.9 \mathrm{TeV}$ in the electron and muon channels, respectively.

\section{Background}

Searching for deviations from the steeply falling $M_{\mathrm{T}}$ spectrum requires an accurate background estimate at very high transverse masses. For the majority of background sources, the estimate is determined from simulation, based on samples with large event counts at high $M_{\mathrm{T}}$. The primary source of background for all signals is the presence of off-peak, high transverse mass tails of the SM W $\rightarrow \ell \nu$ decays. Other backgrounds arise from multijet events from QCD processes, $t \bar{t}$ and single top quark production, and from Drell-Yan events. Contributions from dibosons (WW, WZ, ZZ) decaying to e or $\mu$ are also considered.

The dominant and irreducible background is $\mathrm{W} \rightarrow \ell \nu$ with $\ell=\mathrm{e}, \mu$, and $\tau$. The $\mathrm{W} \rightarrow \tau \nu$ process mostly contributes to the region of lower $M_{\mathrm{T}}$ values in comparison to decays into the other lepton channels, because of the large fraction of the momentum carried away by the two neutrinos from the tau lepton decay. To estimate the dominant $\mathrm{SM} \mathrm{W}$ boson background, several different $\mathrm{W} \rightarrow \ell \nu$ samples are used: one is generated at next-to-leading order (NLO) by MADGRAPH5_aMC@NLO [41] with the NNPDF3.0 PDF set [42], describing the events with an off-shell $\mathrm{W}$ boson mass up to $100 \mathrm{GeV}$, and additional 

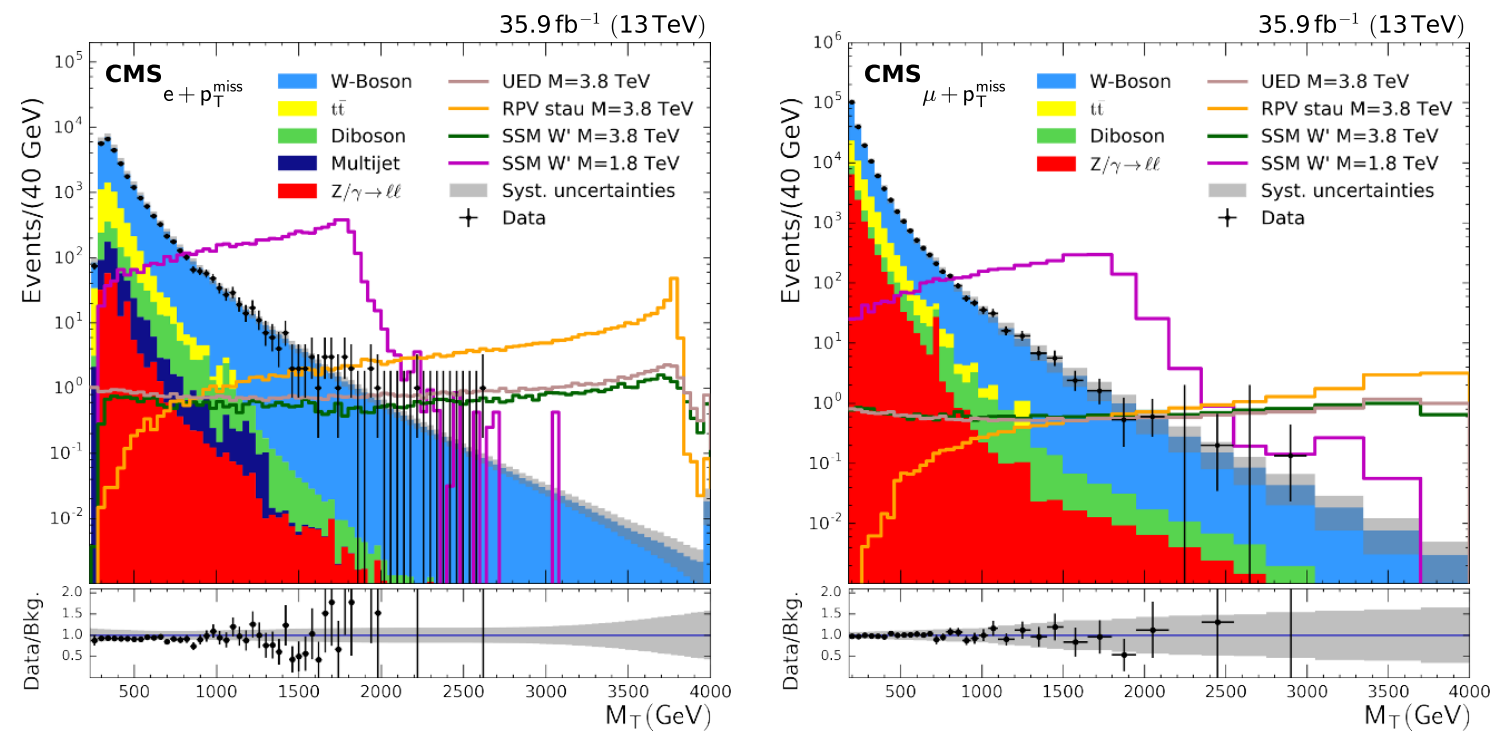

Figure 3. Observed $M_{\mathrm{T}}$ distributions for the electron (left) and muon (right) channels after all selections. Signal examples for $\mathrm{W}^{\prime}$ masses of 1.8 and $3.8 \mathrm{TeV}$, including detector simulation, are shown in both channels. In addition, signal examples for RPV SUSY and split-UED are depicted. The lower panel shows the ratio of data to prediction and the hatched band includes the systematic uncertainties. The last bin shows the total overflow.

\begin{tabular}{|ccccc|}
\hline & $M_{\mathrm{T}}>1 \mathrm{TeV}$ & $M_{\mathrm{T}}>2 \mathrm{TeV}$ & $M_{\mathrm{T}}>3 \mathrm{TeV}$ & $M_{\mathrm{T}}>4 \mathrm{TeV}$ \\
\hline Electron data & 200 & 2 & 0 & 0 \\
Sum of SM backgrounds & $213 \pm 28$ & $5.00 \pm 0.96$ & $0.260 \pm 0.077$ & $0.0163 \pm 0.0078$ \\
SSM W' $\mathrm{M}=1.8 \mathrm{TeV}$ & $5040 \pm 770$ & $25.9 \pm 5.8$ & $0.43 \pm 0.44$ & $0 \pm 0$ \\
$\mathrm{M}=2.4 \mathrm{TeV}$ & $1180 \pm 200$ & $560 \pm 100$ & $1.14 \pm 0.44$ & $0 \pm 0$ \\
$\mathrm{M}=3.8 \mathrm{TeV}$ & $53 \pm 13$ & $40 \pm 11$ & $23.9 \pm 8.4$ & $0.44 \pm 0.25$ \\
$\mathrm{M}=4.2 \mathrm{TeV}$ & $23.3 \pm 7.3$ & $17.6 \pm 6.5$ & $11.8 \pm 5.4$ & $3.4 \pm 2.2$ \\
& 208 & 4 & 0 & 0 \\
Muon data & $217 \pm 20$ & $6.0 \pm 1.2$ & $0.27 \pm 0.21$ & $0.02 \pm 0.02$ \\
Sum of $\mathrm{SM}$ backgrounds & $5345 \pm 530$ & $96 \pm 14$ & $2.5 \pm 1.2$ & $0 \pm 0$ \\
SSM W' $\mathrm{M}=1.8 \mathrm{TeV}$ & $537.4 \pm 5$ & $2.4 \pm 1.2$ & $0.10 \pm 0.05$ \\
$\mathrm{M}=2.4 \mathrm{TeV}$ & $1282 \pm 120$ & $577 \pm 85$ & $24 \pm 12$ & $2 \pm 1$ \\
$\mathrm{M}=3.8 \mathrm{TeV}$ & $57 \pm 6$ & $42 \pm 6$ & $12 \pm 6$ & $3.6 \pm 1.8$ \\
$\mathrm{M}=4.2 \mathrm{TeV}$ & $25 \pm 3$ & $19 \pm 3$ & & \\
\hline
\end{tabular}

Table 1. Expected and observed numbers of signal and background events, for a selected set of $M_{\mathrm{T}}$ thresholds. Also shown are the total systematic uncertainties in the estimate of the event numbers. The signal yields are based on NNLO cross sections. 
samples, covering the boson high-mass region (from $100 \mathrm{GeV}$ onwards), are generated at LO with PYTHiA 8.212, tune CUETP8M1 and NNPDF2.3 PDF. Higher-order electroweak (EW) and QCD multijet corrections are evaluated in bins of $M_{\mathrm{T}}$, following the procedure used in a previous CMS publication [9]. They are calculated using FEWz $3.1[29,30]$ at NNLO QCD multijet precision and MCSANC 1.01 [43] at NLO electroweak precision. The resulting K-factors depend on $M_{\mathrm{T}}$, being around 1.1 for $M_{\mathrm{T}}=400 \mathrm{GeV}$ and decreasing to around 0.8 for $M_{\mathrm{T}}=3 \mathrm{TeV}$.

Top quark pair and single top quark production are other sources of high- $p_{\mathrm{T}}$ leptons and $p_{\mathrm{T}}^{\text {miss }}$, and these are generated with POWHEG 2.0 [44-47] in combination with PYTHIA 8.212, except for the $s$-channel of single top quark production, which is generated

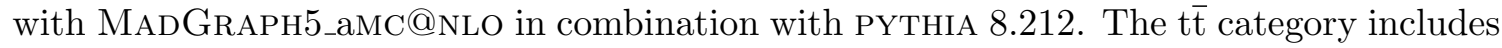
both semileptonic and dileptonic decay modes samples. A NNLO cross section calculation from ref. [48] is used to rescale the NLO predictions. These events are largely rejected by requiring compatibility with a two body decay, but the remaining events can extend into the region of high $M_{\mathrm{T}}$, as seen in figure 3 .

Drell-Yan production of dileptons $(\ell=\mathrm{e}, \mu)$ constitutes a background when one lepton escapes detection. High mass Drell-Yan samples are generated with POWHEG at NLO, with parton showering and hadronization described by PYTHIA, using the CUETP8M1 tune and NNPDF3.0 PDF set.

Contributions from dibosons are derived from inclusive samples (WZ, ZZ, including all possible final states), generated with PYTHIA 8.2.1.2 with the tune CUETP8M1 and the NNPDF2.3 LO PDF set, and exclusive samples (WW $\rightarrow \ell \nu \mathrm{qq}, \mathrm{WW} \rightarrow 4 \mathrm{q}, \mathrm{WW} \rightarrow \ell \ell \nu \nu$, with $\ell=\mathrm{e}, \mu, \tau)$, generated with POWHEG. The inclusive (exclusive) normalizations are scaled to NLO (NNLO) cross sections [49-51].

In the electron channel, a $\gamma+$ jet event sample, generated with MADGRAPH 5_MLM [52] at LO, is used to estimate the effects of photons misidentified as electrons.

The misidentification of jets as leptons is a possible source of background for this search. While the contribution of QCD multijet events to the muon channel is negligible, a small contribution to the electron channel remains after event selection. For the latter, the shape and normalization of the QCD multijet background is derived from data and included in the final background estimate shown in figure 3 .

A QCD multijet template is obtained from the events in which the electron candidate fails the isolation requirement but where all other event requirements are met. QCD multijet template events are scaled with normalization factors from an independent control region, defined by the requirement $p_{\mathrm{T}} / p_{\mathrm{T}}^{\text {miss }}>1.5$, where multijet production is dominating. In this region, the ratio of 'tight' events (electron candidates that passes all requirements of a well-isolated electron) to 'loose' events (all events in the region) is measured as a function of electron $p_{\mathrm{T}}$. The resulting normalization factor for QCD multijet template events is applied to non-QCD subtracted data. This procedure results in ratios from about $10 \%$ for $p_{\mathrm{T}}$ $=200 \mathrm{GeV}$ down to $1 \%$ for $p_{\mathrm{T}}>600 \mathrm{GeV}$, and represents the percentage of jets that are misreconstructed as electrons. An uncertainty of $40 \%$ in this estimate is obtained by comparing data to predictions from simulation, and is assigned to this small background contribution. 


\section{Systematic uncertainties}

The mismeasurement of lepton energy or momentum, arising from both detector resolution and imperfect scale calibration, will result in a smearing of the $M_{\mathrm{T}}$ spectrum. For each source of uncertainty, shifts of $\pm 1 \sigma$ are applied to the simulated data. The kinematic distributions of the objects $\left(\mathrm{e}, \mu, p_{\mathrm{T}}^{\mathrm{miss}}\right)$ and $M_{\mathrm{T}}$ are recalculated, and the kinematic selection is reapplied.

The systematic uncertainty in the electron energy scale is estimated to be $0.4(0.8) \%$ in the barrel (endcaps). For the electron energy resolution uncertainty, an additional Gaussian smearing of $1.2(2.4) \%$ for the barrel (endcap) region is applied to the simulation [53] to reflect the observed behaviour of the calorimeter.

The muon transverse momentum scale uncertainty is estimated by studying the curvature of muon tracks in different regions of $\eta$ and $\phi$, using high- $p_{\mathrm{T}}$ muons from cosmic ray data [38] and dimuon events from high- $p_{\mathrm{T}} \mathrm{Z}$ boson decays from collision data, together with corresponding simulation samples. These studies indicate the absence of a significant curvature bias in the central $\eta$ region $(|\eta|<1.2)$, within an uncertainty of $0.025 / \mathrm{TeV}$. In more forward regions, especially for $|\eta|>2.1$, and in particular muon $\phi$ zones, values of the scale bias different from zero are found. The mean value of the modification in the simulated $p_{\mathrm{T}}$ coming from these scale biases is used as an estimate of the uncertainty, for $p_{\mathrm{T}}>200 \mathrm{GeV}$. This uncertainty is propagated to $p_{\mathrm{T}}^{\text {miss }}$ and $M_{\mathrm{T}}$.

The uncertainty in the muon momentum measurement derives from the smallness of curvature of tracks for high- $p_{\mathrm{T}}$ muons, while the energy of the electrons, measured in the crystal calorimeter, has a smaller uncertainty. The muon $p_{\mathrm{T}}$ resolution in data at high- $p_{\mathrm{T}}$ values is well reproduced by the simulation within an uncertainty of $\pm 1(2) \%$ in the barrel (endcap) region and no additional smearing is implemented.

The overall uncertainty in the determination of $p_{\mathrm{T}}^{\text {miss }}$ in each event is derived from the individual uncertainties assigned to the objects (jets, e, $\mu, \tau, \gamma$, and unclustered energy) obtained from the PF algorithm. The contribution of each object type is varied according to its uncertainty. In addition, an uncertainty of $10 \%$ in the $p_{\mathrm{T}}$ is used for the unclustered energy. This uncertainty is propagated to the $\mathrm{PF} p_{\mathrm{T}}^{\text {miss }}$ [35]. The quadratic sum of the individual uncertainties gives the overall uncertainty in the $\mathrm{PF} p_{\mathrm{T}}^{\text {miss }}$.

In addition to the cross section uncertainties, two further contributions play a role in the background prediction uncertainties. For the dominant $\mathrm{W}$ boson production, the uncertainties associated with the method chosen to calculate the K-factor are considered, amounting to $\pm 5 \%$. The effect of even higher order corrections is expected to be small and therefore is not considered. For the QCD multijet background prediction, an uncertainty of $40 \%$ is used as described in section 6 .

The theoretical uncertainty related to the choice of PDF set was estimated using the PDF4LHC recommended procedure [54]. After reweighting the background samples with different PDF sets, the envelopes of their error bands are taken as the uncertainty. The values increase with $M_{\mathrm{T}}$, ranging from an uncertainty of $1 \%$ at $M_{\mathrm{T}}=300 \mathrm{GeV}$ to $20 \%$ at $M_{\mathrm{T}} \approx 3 \mathrm{TeV}$. 
The efficiency scale factors defined in section 4 are assigned uncertainties that take into account the determination method and the extrapolation to high $M_{\mathrm{T}}$.

The simulated distribution of pp collision vertices per bunch crossing is reweighted to the distribution measured in data. The uncertainty due to this reweighting method is treated as the systematic uncertainty of the pileup simulation. The effect on the background event yield from this uncertainty is smaller than $5 \%$.

The estimated uncertainty in the integrated luminosity measurement is $2.5 \%$ [55].

\section{Interpretation of the results}

No significant deviation from the SM expectation is observed in either channel and exclusion limits on the production cross sections of the theoretical models from section 3 are calculated. All limits presented here are at 95\% CL.

The signal search is performed using the binned $M_{\mathrm{T}}$ distribution, obtained after the complete event selection, taking into account the shape and normalization of the signal and backgrounds. As shown in figure 3, signal events are expected to be particularly prominent at the upper end of their $M_{\mathrm{T}}$ distribution, where the expected SM background is low. The final $M_{\mathrm{T}}$ distributions are presented in figure 3 for electron and muon channels, and together with the detailed systematic uncertainties described in section 7 .

\subsection{Statistical analysis}

Upper limits on the product of the production cross section and branching fraction $\sigma_{\mathrm{W}^{\prime}} \mathcal{B}\left(\mathrm{W}^{\prime} \rightarrow \ell \nu\right)$, with $\ell=\mathrm{e}$ or $\mu$, are determined using a Bayesian method [56] with an uniform positive prior probability distribution for the signal cross section. Systematic uncertainties in the expected signal and background yields are included either via nuisance parameters with log normal prior distributions or with the shape of the distribution included through the use of a binned likelihood (multi-bin counting). For the SSM $\mathrm{W}^{\prime}$ and split-UED models, the limits are obtained from the entire $M_{\mathrm{T}}$ spectrum for $M_{\mathrm{T}}>220 \mathrm{GeV}$, as displayed in figure 3, using the multi-bin counting method. This procedure is performed for different values of parameters of each signal, to obtain limits in terms on these parameters, such as the $\mathrm{W}^{\prime}$ boson mass.

To determine a model-independent upper limit on the product of the cross section and branching fraction, all events above a threshold $M_{\mathrm{T}}^{\mathrm{min}}$ are summed. From the number of background events, signal events, and observed data events, the cross section limit can be calculated. When the background is low, this method has good sensitivity, comparable to that of the multi-bin approach. The resulting limit can be reinterpreted for other models with a lepton and $p_{\mathrm{T}}^{\text {miss }}$ in the final state. One example application is given in this paper, where limits on specific RPV SUSY processes are derived.

\subsection{Model-independent cross section limit}

A model-independent cross section limit is determined using a single bin ranging from a lower threshold on $M_{\mathrm{T}}$ to infinity. No assumptions on the shape of the signal $M_{\mathrm{T}}$ distribution have to be made other than that of a flat product $A \epsilon$ of acceptance and 

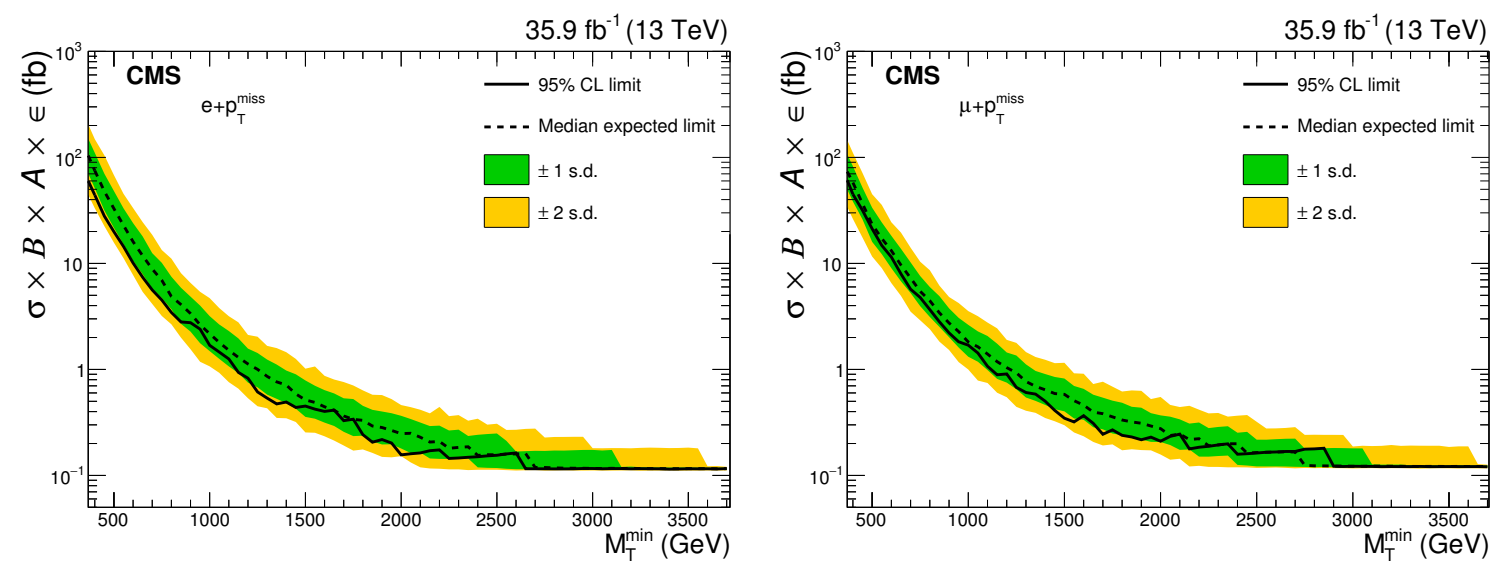

Figure 4. Cross section upper limits at 95\% CL on the effective cross section $\sigma\left(\mathrm{W}^{\prime}\right) \mathcal{B}\left(\mathrm{W}^{\prime} \rightarrow \ell \nu\right) A \epsilon$ above a threshold $M_{\mathrm{T}}^{\min }$ for the individual electron (left) and muon (right) channels. Shown are the observed limit (solid line), expected limit (dashed line), and the expected limit \pm 1 and \pm 2 standard deviation (s.d.) intervals.

efficiency as a function of $\mathrm{W}^{\prime}$ mass. In order to determine any limit for a specific model from the model-independent limit shown here, only the model-dependent part of the efficiency needs to be applied. The experimental efficiencies for the signal are already taken into account, including the effect of the kinematic selection (the cuts on $p_{\mathrm{T}} / p_{\mathrm{T}}^{\mathrm{miss}}$ and $\Delta \phi$ ), the geometrical acceptance (cut on $\eta$ ), and the trigger threshold.

A factor $f_{M_{\mathrm{T}}}$ that reflects the effect of the threshold $M_{\mathrm{T}}^{\mathrm{min}}$ on the signal is determined by counting the events with $M_{\mathrm{T}}>M_{\mathrm{T}}^{\min }$ and dividing it by the number of generated events. For $M_{\mathrm{T}}>400 \mathrm{GeV}$ the reconstruction efficiency is nearly constant, therefore $f_{M_{\mathrm{T}}}$ can be evaluated at generator level. For lower $M_{\mathrm{T}}$, a very slight $(<1 \%)$ difference is expected due to the single-lepton trigger threshold (in particular the $130 \mathrm{GeV}$ threshold for electrons). A limit on the product of the cross section and branching fraction $(\sigma \mathcal{B} A \epsilon)_{\text {excl }}$ can be obtained by dividing the excluded cross section of the model-independent limit $(\sigma \mathcal{B} A \epsilon)_{\mathrm{MI}}$ given in figure 4 by the calculated fraction $f_{M_{\mathrm{T}}}\left(M_{\mathrm{T}}^{\mathrm{min}}\right)$ :

$$
(\sigma \mathcal{B} A \epsilon)_{\mathrm{excl}}=\frac{(\sigma \mathcal{B} A \epsilon)_{\mathrm{MI}}\left(M_{\mathrm{T}}^{\mathrm{min}}\right)}{f_{M_{\mathrm{T}}}\left(M_{\mathrm{T}}^{\mathrm{min}}\right)}
$$

Models with a theoretical cross section $(\sigma \mathcal{B})_{\text {theo }}$ larger than $(\sigma \mathcal{B})_{\text {excl }}$ can be excluded. The procedure described here can be applied to models that exhibit back-to-back kinematics similar to those of a generic $\mathrm{W}^{\prime}$, which is a reasonable assumption for a two-body decay of a massive state. If the kinematic properties are different, the fraction of events $f_{M_{\mathrm{T}}}\left(M_{\mathrm{T}}^{\mathrm{min}}\right)$ needs to be determined for the model considered. The results for the electron and muon channels are shown separately in figure 4 . The results depend strongly on the threshold $M_{\mathrm{T}}^{\mathrm{min}}$. In both channels, cross section values $\sigma B A \epsilon$ between $40-50 \mathrm{fb}$ (for $M_{\mathrm{T}}^{\min }>$ $400 \mathrm{GeV})$ and $0.1 \mathrm{fb}\left(M_{\mathrm{T}}^{\min }>3 \mathrm{TeV}\right)$ are excluded for the $M_{\mathrm{T}}^{\min }$ thresholds given in brackets. 

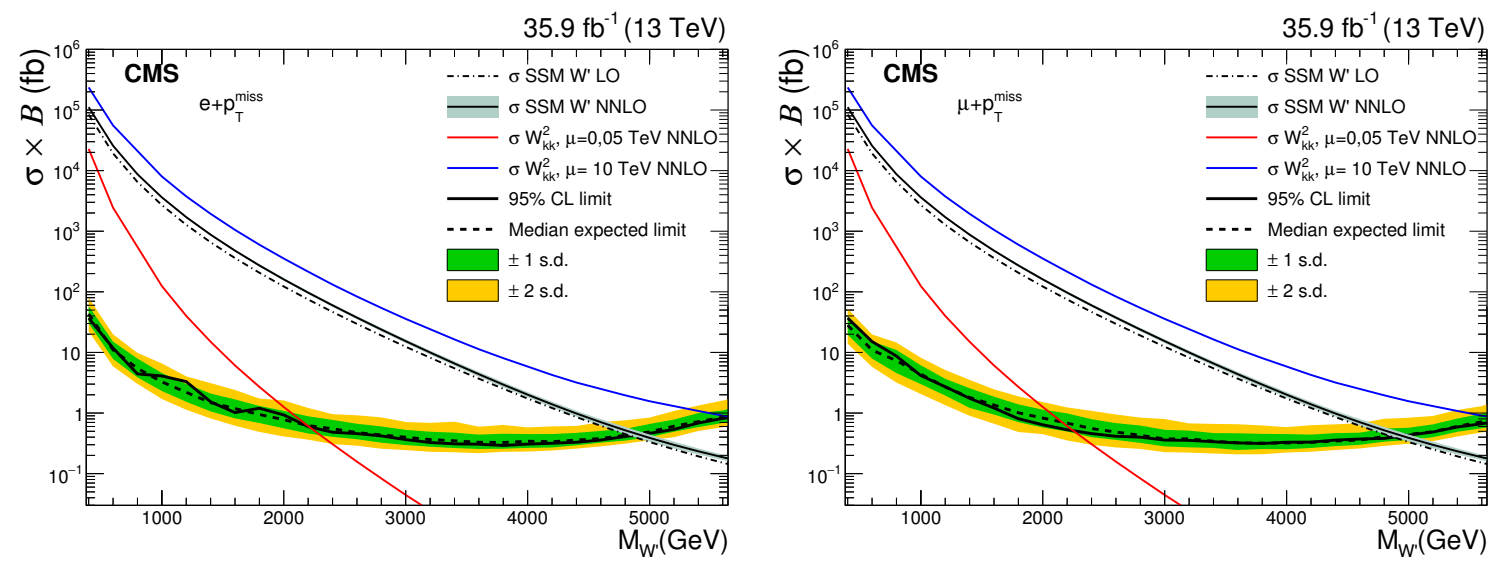

Figure 5. Expected (dashed line) and observed (solid line) 95\% CL limits in the SSM interpretation for the electron (left) and muon (right) channels. The shaded bands represent the one and two standard deviation (s.d.) uncertainty bands. Also shown are theoretical cross sections for the SSM benchmark model (black with a grey band for the PDF uncertainties) and split-UED (red and blue solid lines) interpretations.

\subsection{Limits on an SSM $\mathrm{W}^{\prime}$ boson}

The search for an SSM $\mathrm{W}^{\prime}$ boson yields limits on the product of the cross section and branching fraction for the electron and muon channels. The multi-bin method is used to determine the 95\% CL upper cross section limits, shown in figure 5. The indicated theoretical cross sections are the NNLO values for the lepton $+p_{\mathrm{T}}^{\text {miss }}$ channel, as detailed in section 3, and are the same for both channels. The PDF uncertainties are shown as a thin band around the NNLO cross section. The central value of the product of the theoretical cross section and branching fraction is used for deriving the mass limit. Values of the $\mathrm{W}^{\prime}$ mass below $4.9 \mathrm{TeV}$ (expected limit is $5.0 \mathrm{TeV}$ ) and $4.9 \mathrm{TeV}$ (expected limit is $4.9 \mathrm{TeV}$ ) are excluded in the electron and muon channel, respectively. Also shown in figure 5 are theoretical cross sections for a number of interpretations. For the benchmark SSM model, the displayed NNLO theoretical cross section includes the branching fraction to an electron or muon, as appropriate.

When combining both channels, the exclusion limit increases to $5.2 \mathrm{TeV}$ (expected limit is $5.2 \mathrm{TeV}$ ), as depicted in figure 6 . This is a significant improvement over the previous $13 \mathrm{TeV}$ result [9] of $3.6 \mathrm{TeV}$ and $3.9 \mathrm{TeV}$ in the electron and muon channels, respectively. For high $\mathrm{W}^{\prime}$ masses (around $5 \mathrm{TeV}$ and higher), the mass limit becomes less stringent because of the increasing fraction of off-shell production. For high masses, the search sensitivity is limited by the amount of data available at present and will improve in future. The one and two standard deviation bands in the figures represent the systematic uncertainties as described in section 7 .

In addition, the LO cross section for the SSM model is shown. For the split-UED model, NNLO cross sections are displayed for two extreme values of the bulk mass parameter $\mu$ corresponding to a $\mu=0.05 \mathrm{TeV}$ (boson mass limit of $2.4 \mathrm{TeV}$ ) and a $\mu=10 \mathrm{TeV}$ (boson mass limit of $5.6 \mathrm{TeV})$. 

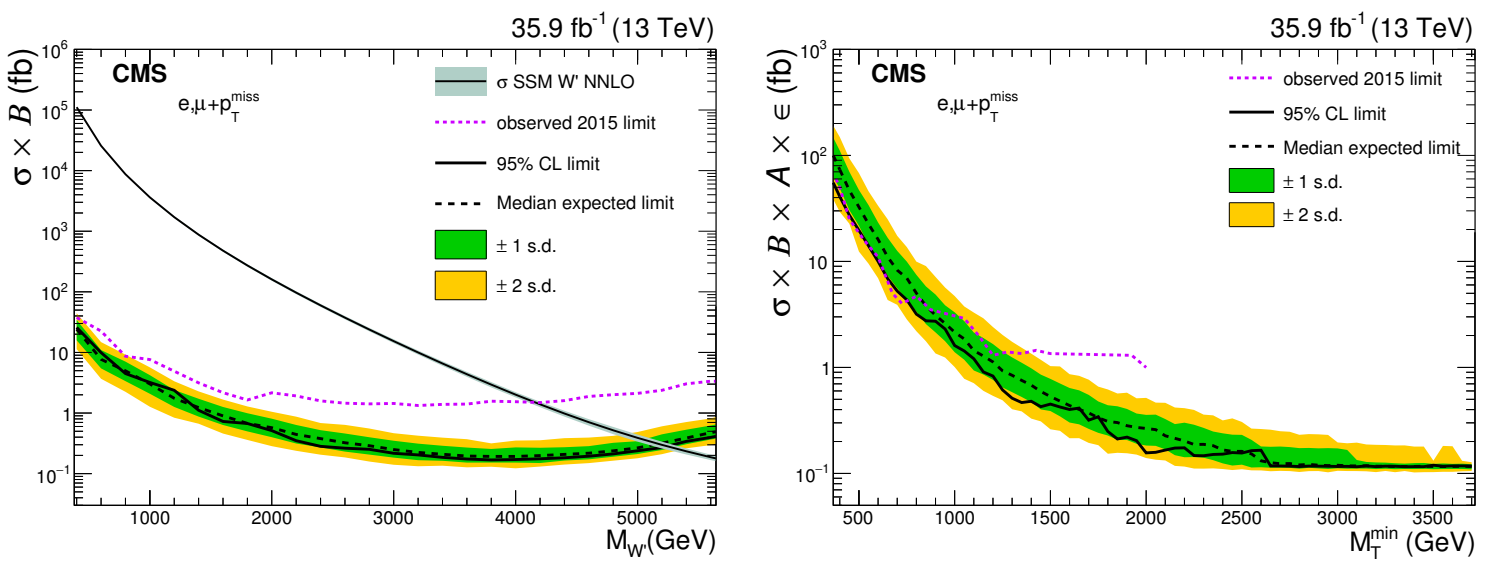

Figure 6. Expected (dashed line) and observed (solid line) 95\% CL limits on the product of the cross section and branching fraction, in the SSM $\mathrm{W}^{\prime}$ model (left) and the model-independent interpretation (right). Shown are the combination of the electron and muon channels, with the shaded bands corresponding to one and two standard deviations (s.d.). For comparison, the results from ref. [9] for the regions investigated in 2015 are shown as dotted lines.

\subsection{Limits on the coupling strength}

The limit on the cross section depends on both the mass range of a potential excess and the width. Because of the relation between the coupling of a particle and its width, a limit can also be set on the coupling strength.

In order to compute the limit for couplings other than $g_{\mathrm{W}^{\prime}} / g_{\mathrm{W}} \neq 1$, reweighted samples are used that take into account the appropriate signal width and the differences in reconstructed $M_{\mathrm{T}}$ shapes. For $g_{\mathrm{W}^{\prime}} / g_{\mathrm{W}}=1$ the theoretical LO cross sections apply.

Based on the $M_{\mathrm{T}}$ distribution of data after all selections, shown in figure 3 , a cross section limit as a function of the coupling is determined for each mass point. The procedure is repeated for the full range of $\mathrm{W}^{\prime}$ masses and the corresponding intersection points provide the input for the exclusion limit on the coupling strength $g_{\mathrm{W}^{\prime}} / g_{\mathrm{W}}$ as a function of the $\mathrm{W}^{\prime}$ mass, as shown in figure 7. Everything above the experimental limit is excluded. For low masses, weak couplings down to nearly $10^{-2}$ are excluded.

\subsection{Interpretation in the split-UED model}

The UED model is parameterized by the quantities $R$ and $\mu$, which are the radius of the extra dimension and the bulk mass parameter of the fermion field in five dimensions. The lower limits on the mass for $n=2$ can be directly translated from the SSM $W^{\prime}$ limit into bounds on the split-UED parameter space $(1 / R, \mu)$, as the signal shape and signal efficiency are identical to the SSM $W^{\prime}$ signal. The split-UED limits are displayed in figure 8, separately for each channel as well as the combination. The observed experimental limits on the inverse radius of the extra dimension $R$ are $2.8 \mathrm{TeV}$ (electrons) and $2.75 \mathrm{TeV}$ (muons) at $\mu \approx 10 \mathrm{TeV}$. For the combination, a limit on $1 / R$ of $2.9 \mathrm{TeV}$ is obtained for $\mu \geq 4 \mathrm{TeV}$. For comparison, the observed limit on $1 / R$ from the $8 \mathrm{TeV}$ analysis [8] based on the combination of electron and muon channels is $1.8 \mathrm{TeV}$. 

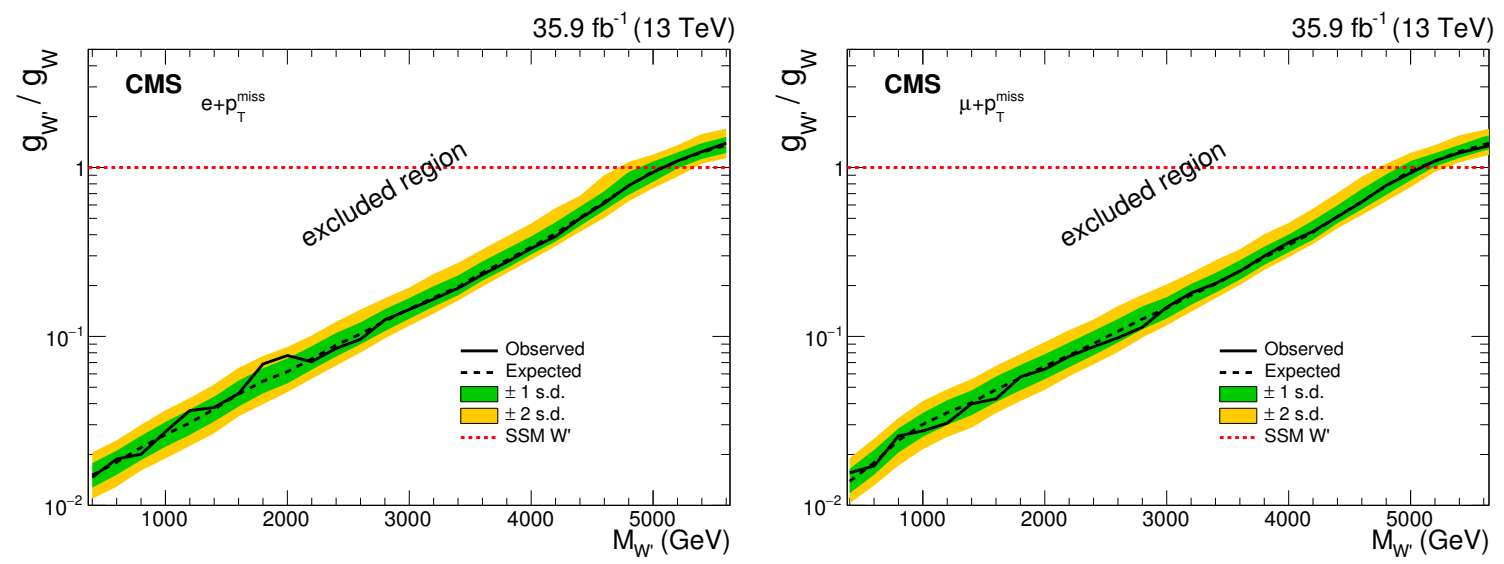

Figure 7. Expected (dashed line) and observed (solid line) 95\% CL limits on the coupling strength $g_{\mathrm{W}^{\prime}} / g_{\mathrm{W}}$ as a function of the $\mathrm{W}^{\prime}$ mass, for the electron (left) and muon (right) channels. The area above the limit line is excluded. The shaded bands represent the one and two standard deviation (s.d.) uncertainty bands. The SSM $\mathrm{W}^{\prime}$ couplings are shown as a dotted line.
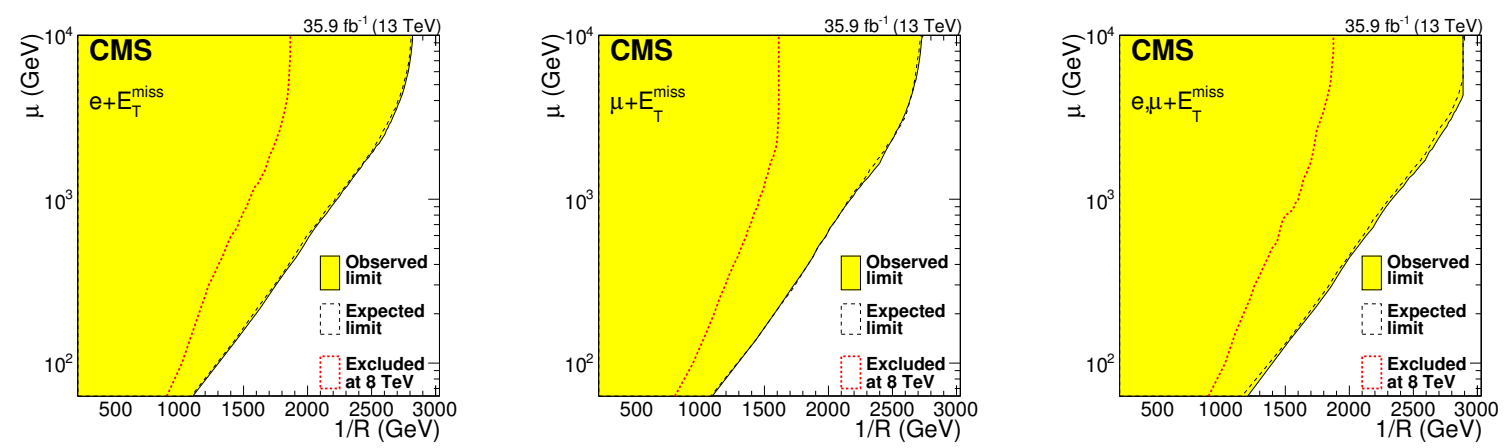

Figure 8. Exclusion limit in the plane $(\mu, 1 / \mathrm{R})$ for the split-UED interpretation for the $n=2$ case in the electron (left) and muon (middle) channels, respectively. On the right, the result for the combination of both channels is shown. The expected limit is depicted as a dashed line and the experimentally excluded region as a solid black line (filled as a yellow area). For comparison, the $8 \mathrm{TeV}$ result from ref. [8] is given as a red, dotted line.

\subsection{Limits on RPV SUSY}

An alternative interpretation assumes a $\tau$ slepton as a mediator, with distinct RPV SUSY couplings at the production and decay vertices, as detailed in section 3.4. This result is obtained using the model-independent limit, and illustrates its power. The couplings are defined in figure 1. While $\lambda_{3 i j}^{\prime}$ is common to both decay channels, the coupling at the decay is either $\lambda_{231}$ (for $\mathrm{e}+\nu_{\mu}$ ) or $\lambda_{132}$ (for $\mu+\nu_{\mathrm{e}}$ ). This signal has a slightly different shape compared to the SSM W', a sharper Jacobian peak and essentially no off-shell part at high mediator masses affecting the signal efficiency. In particular at low masses, the tau slepton signal efficiency is higher. Applying the procedure from section 8.2, the fraction of events $f_{M_{\mathrm{T}}}$ is determined for the $\tau$ slepton model at generator level and a correction with respect to the SSM $\mathrm{W}^{\prime}$ is applied. For a $\tau$ slepton mass of $3.6 \mathrm{TeV}$, as a function of 

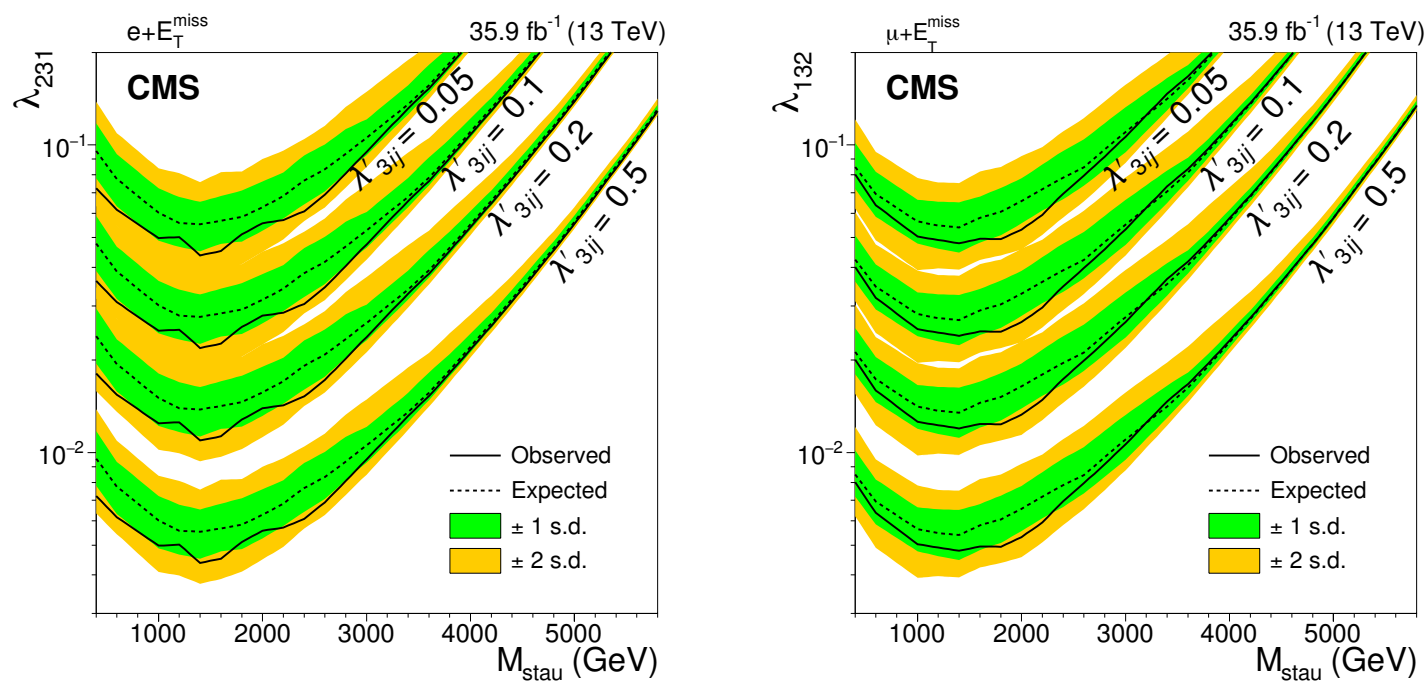

Figure 9. Observed (solid line) and expected (dashed line) exclusion limits for different couplings in the model with a $\tau$ slepton as a mediator. The couplings $\lambda_{3 i j}^{\prime}, \lambda_{231}$, and $\lambda_{132}$ are defined in figure 1 . Results are shown for the final states consisting of $\mathrm{e}+\nu_{\mu}$ on the left and $\mu+\nu_{\mathrm{e}}$ on the right.

$M_{\mathrm{T}}^{\min }$, representative values are: $f_{M_{\mathrm{T}}}$ is $0.99\left(400 \mathrm{GeV}<M_{\mathrm{T}}^{\min }<750 \mathrm{GeV}\right), 0.90\left(M_{\mathrm{T}}^{\min }=\right.$ $1650 \mathrm{GeV})$, and $0.82\left(M_{\mathrm{T}}^{\min }=2100 \mathrm{GeV}\right)$. Exclusion limits on $\lambda_{231}$ and $\lambda_{123}$ as a function of the mediator mass for a number of coupling values $\lambda_{3 i j}^{\prime}$ are shown in figure 9 .

\section{Summary}

A search for high-mass resonances in the lepton plus the missing transverse momentum final state in proton-proton collisions at $\sqrt{s}=13 \mathrm{TeV}$ has been performed, using a data sample corresponding to an integrated luminosity of $35.9 \mathrm{fb}^{-1}$. No evidence for new physics is observed when examining the transverse mass distributions in the electron and muon channels. These observations are interpreted as $95 \%$ confidence limits on the parameters of several models.

The exclusion limits on a sequential standard model-like $\mathrm{W}^{\prime}$ are calculated to be 4.9 (4.9) $\mathrm{TeV}$ in the individual electron (muon) channels. A combination of both channels increases the limit to $5.2 \mathrm{TeV}$, assuming standard model couplings. Additionally, variations in the coupling strength are studied and couplings above $2 \times 10^{-2}$ are excluded for low $\mathrm{W}^{\prime}$ masses. These results are also applied to the split universal extra dimension model, and the inverse radius of the extra dimension $1 / R$ is constrained by this analysis to be above $2.9 \mathrm{TeV}$.

These results are presented in a model-independent form, making possible their interpretation in a number of other models. An example of this application is given using a supersymmetric model with R-parity violation, and a tau slepton mediator with flavorviolating decays into either $\mathrm{e}+\nu_{\mu}$ or $\mu+\nu_{\mathrm{e}}$. Limits on the coupling strengths at the decay vertex have been derived as a function of the mediator mass, for various values of the coupling at the production vertex $\lambda_{3 i j}^{\prime}$. 


\section{Acknowledgments}

We congratulate our colleagues in the CERN accelerator departments for the excellent performance of the LHC and thank the technical and administrative staffs at CERN and at other CMS institutes for their contributions to the success of the CMS effort. In addition, we gratefully acknowledge the computing centers and personnel of the Worldwide LHC Computing Grid for delivering so effectively the computing infrastructure essential to our analyses. Finally, we acknowledge the enduring support for the construction and operation of the LHC and the CMS detector provided by the following funding agencies: BMWFW and FWF (Austria); FNRS and FWO (Belgium); CNPq, CAPES, FAPERJ, and FAPESP (Brazil); MES (Bulgaria); CERN; CAS, MoST, and NSFC (China); COLCIENCIAS (Colombia); MSES and CSF (Croatia); RPF (Cyprus); SENESCYT (Ecuador); MoER, ERC IUT, and ERDF (Estonia); Academy of Finland, MEC, and HIP (Finland); CEA and CNRS/IN2P3 (France); BMBF, DFG, and HGF (Germany); GSRT (Greece); NKFIA (Hungary); DAE and DST (India); IPM (Iran); SFI (Ireland); INFN (Italy); MSIP and NRF (Republic of Korea); LAS (Lithuania); MOE and UM (Malaysia); BUAP, CINVESTAV, CONACYT, LNS, SEP, and UASLP-FAI (Mexico); MBIE (New Zealand); PAEC (Pakistan); MSHE and NSC (Poland); FCT (Portugal); JINR (Dubna); MON, RosAtom, RAS, RFBR and RAEP (Russia); MESTD (Serbia); SEIDI, CPAN, PCTI and FEDER (Spain); Swiss Funding Agencies (Switzerland); MST (Taipei); ThEPCenter, IPST, STAR, and NSTDA (Thailand); TUBITAK and TAEK (Turkey); NASU and SFFR (Ukraine); STFC (United Kingdom); DOE and NSF (U.S.A.).

Individuals have received support from the Marie-Curie program and the European Research Council and Horizon 2020 Grant, contract No. 675440 (European Union); the Leventis Foundation; the A. P. Sloan Foundation; the Alexander von Humboldt Foundation; the Belgian Federal Science Policy Office; the Fonds pour la Formation à la Recherche dans l'Industrie et dans l'Agriculture (FRIA-Belgium); the Agentschap voor Innovatie door Wetenschap en Technologie (IWT-Belgium); the F.R.S.-FNRS and FWO (Belgium) under the "Excellence of Science - EOS" - be.h project n. 30820817; the Ministry of Education, Youth and Sports (MEYS) of the Czech Republic; the Lendület ("Momentum") Program and the János Bolyai Research Scholarship of the Hungarian Academy of Sciences, the New National Excellence Program ÚNKP, the NKFIA research grants 123842, 123959, 124845, 124850 and 125105 (Hungary); the Council of Science and Industrial Research, India; the HOMING PLUS program of the Foundation for Polish Science, cofinanced from European Union, Regional Development Fund, the Mobility Plus program of the Ministry of Science and Higher Education, the National Science Center (Poland), contracts Harmonia 2014/14/M/ST2/00428, Opus 2014/13/B/ST2/02543, 2014/15/B/ST2/03998, and 2015/19/B/ST2/02861, Sonata-bis 2012/07/E/ST2/01406; the National Priorities Research Program by Qatar National Research Fund; the Programa Estatal de Fomento de la Investigación Científica y Técnica de Excelencia María de Maeztu, grant MDM-2015-0509 and the Programa Severo Ochoa del Principado de Asturias; the Thalis and Aristeia programs cofinanced by EU-ESF and the Greek NSRF; the Rachadapisek Sompot Fund for Postdoctoral Fellowship, Chulalongkorn University and the Chulalongkorn Academic into 
Its 2nd Century Project Advancement Project (Thailand); the Welch Foundation, contract C-1845; and the Weston Havens Foundation (U.S.A.).

Open Access. This article is distributed under the terms of the Creative Commons Attribution License (CC-BY 4.0), which permits any use, distribution and reproduction in any medium, provided the original author(s) and source are credited.

\section{References}

[1] K. Hsieh, K. Schmitz, J.-H. Yu and C.P. Yuan, Global analysis of general $\mathrm{SU}(2) \times \mathrm{SU}(2) \times \mathrm{U}(1)$ models with precision data, Phys. Rev. D 82 (2010) 035011 [arXiv: 1003.3482] [INSPIRE].

[2] N. Arkani-Hamed, S. Dimopoulos and G.R. Dvali, The hierarchy problem and new dimensions at a millimeter, Phys. Lett. B 429 (1998) 263 [hep-ph/9803315] [INSPIRE].

[3] T. Appelquist, H.-C. Cheng and B.A. Dobrescu, Bounds on universal extra dimensions, Phys. Rev. D 64 (2001) 035002 [hep-ph/0012100] [InSPIRE].

[4] G. Altarelli, B. Mele and M. Ruiz-Altaba, Searching for new heavy vector bosons in $p \bar{p}$ colliders, Z. Phys. C 45 (1989) 109 [Erratum ibid. C 47 (1990) 676] [InSPIRE].

[5] H.K. Dreiner and T. Stefaniak, Bounds on R-parity violation from resonant slepton production at the LHC, Phys. Rev. D 86 (2012) 055010 [arXiv: 1201.5014] [InSPIRE].

[6] G.R. Farrar and P. Fayet, Phenomenology of the production, decay and detection of new hadronic states associated with supersymmetry, Phys. Lett. B 76 (1978) 575 [INSPIRE].

[7] K. Kong, S.C. Park and T.G. Rizzo, Collider phenomenology with split-UED, JHEP 04 (2010) 081 [arXiv: 1002.0602] [INSPIRE].

[8] CMS collaboration, Search for physics beyond the Standard Model in final states with a lepton and missing transverse energy in proton-proton collisions at $\sqrt{s}=8 \mathrm{TeV}$, Phys. Rev. D 91 (2015) 092005 [arXiv:1408.2745] [INSPIRE].

[9] CMS collaboration, Search for heavy gauge $W^{\prime}$ boson in events with an energetic lepton and large missing transverse momentum at $\sqrt{s}=13 \mathrm{TeV}$, Phys. Lett. B 770 (2017) 278 [arXiv: 1612.09274] [INSPIRE].

[10] ATLAS collaboration, Search for new particles in events with one lepton and missing transverse momentum in pp collisions at $\sqrt{s}=8 \mathrm{TeV}$ with the ATLAS detector, JHEP 09 (2014) 037 [arXiv: 1407.7494] [INSPIRE].

[11] ATLAS collaboration, Search for a new heavy gauge boson resonance decaying into a lepton and missing transverse momentum in $36 \mathrm{fb}^{-1}$ of pp collisions at $\sqrt{\mathrm{s}}=13 \mathrm{TeV}$ with the ATLAS experiment, Eur. Phys. J. C 78 (2018) 401 [arXiv:1706.04786] [INSPIRE].

[12] CMS collaboration, Performance of photon reconstruction and identification with the CMS detector in proton-proton collisions at $\sqrt{s}=8 \mathrm{TeV}, 2015$ JINST $10 \mathrm{P} 08010$ [arXiv: 1502.02702] [INSPIRE].

[13] CMS collaboration, Search for heavy narrow dilepton resonances in pp collisions at $\sqrt{s}=7 \mathrm{TeV}$ and $\sqrt{s}=8 \mathrm{TeV}$, Phys. Lett. B 720 (2013) 63 [arXiv:1212.6175] [INSPIRE]. 
[14] CMS collaboration, Performance of the CMS muon detector and muon reconstruction with proton-proton collisions at $\sqrt{s}=13$ TeV, 2018 JINST 13 P06015 [arXiv:1804.04528] [INSPIRE].

[15] M. Cacciari, G.P. Salam and G. Soyez, The anti- $k_{t}$ jet clustering algorithm, JHEP 04 (2008) 063 [arXiv: 0802.1189] [INSPIRE].

[16] M. Cacciari, G.P. Salam and G. Soyez, FastJet user manual, Eur. Phys. J. C 72 (2012) 1896 [arXiv:1111.6097] [INSPIRE].

[17] CMS collaboration, The CMS trigger system, 2017 JINST 12 P01020 [arXiv:1609.02366] [INSPIRE].

[18] CMS collaboration, The CMS experiment at the CERN LHC, 2008 JINST 3 S08004 [INSPIRE].

[19] GEANT4 collaboration, S. Agostinelli et al., GEANT4: a simulation toolkit, Nucl. Instrum. Meth. A 506 (2003) 250 [inSPIRE].

[20] D0 collaboration, V.M. Abazov et al., Search for $W^{\prime}$ boson resonances decaying to a top quark and a bottom quark, Phys. Rev. Lett. 100 (2008) 211803 [arXiv:0803.3256] [INSPIRE].

[21] CMS collaboration, Search for heavy resonances decaying to a top quark and a bottom quark in the lepton + jets final state in proton-proton collisions at $13 \mathrm{TeV}$, Phys. Lett. B 777 (2018) 39 [arXiv: 1708.08539] [INSPIRE].

[22] ATLAS collaboration, Search for $W^{\prime} \rightarrow t b \rightarrow q q b b$ decays in pp collisions at $\sqrt{s}=8$ TeV with the ATLAS detector, Eur. Phys. J. C 75 (2015) 165 [arXiv:1408.0886] [INSPIRE].

[23] CMS collaboration, Search for new resonances decaying via $W Z$ to leptons in proton-proton collisions at $\sqrt{s}=8 \mathrm{TeV}$, Phys. Lett. B 740 (2015) 83 [arXiv:1407.3476] [InSPIRE].

[24] CMS collaboration, Search for massive resonances decaying into $W W, W Z$ or $Z Z$ bosons in proton-proton collisions at $\sqrt{s}=13 \mathrm{TeV}$, JHEP 03 (2017) 162 [arXiv:1612.09159] [INSPIRE].

[25] T. Sjöstrand et al., An introduction to PYTHIA 8.2, Comput. Phys. Commun. 191 (2015) 159 [arXiv: 1410.3012] [INSPIRE].

[26] R.D. Ball et al., Impact of heavy quark masses on parton distributions and LHC phenomenology, Nucl. Phys. B 849 (2011) 296 [arXiv:1101.1300] [InSPIRE].

[27] R.D. Ball et al., Parton distributions with LHC data, Nucl. Phys. B 867 (2013) 244 [arXiv:1207.1303] [INSPIRE].

[28] P. Skands, S. Carrazza and J. Rojo, Tuning PYTHIA 8.1: the Monash 2013 tune, Eur. Phys. J. C 74 (2014) 3024 [arXiv:1404.5630] [INSPIRE].

[29] R. Gavin, Y. Li, F. Petriello and S. Quackenbush, FEWZ 2.0: a code for hadronic Z production at next-to-next-to-leading order, Comput. Phys. Commun. 182 (2011) 2388 [arXiv:1011.3540] [INSPIRE].

[30] Y. Li and F. Petriello, Combining QCD and electroweak corrections to dilepton production in FEWZ, Phys. Rev. D 86 (2012) 094034 [arXiv:1208.5967] [InSPIRE].

[31] J. Alwall, M. Herquet, F. Maltoni, O. Mattelaer and T. Stelzer, MadGraph 5: going beyond, JHEP 06 (2011) 128 [arXiv:1106.0522] [INSPIRE].

[32] C.-R. Chen, M.M. Nojiri, S.C. Park, J. Shu and M. Takeuchi, Dark matter and collider phenomenology of split-UED, JHEP 09 (2009) 078 [arXiv:0903.1971] [INSPIRE]. 
[33] CMS collaboration, Particle-flow reconstruction and global event description with the CMS detector, 2017 JINST 12 P10003 [arXiv:1706.04965] [INSPIRE].

[34] CMS collaboration, Performance of missing energy reconstruction in 13 TeV pp collision data using the CMS detector, CMS-JME-16-004, CERN, Geneva, Switzerland, (2016).

[35] CMS collaboration, Missing transverse energy performance of the CMS detector, 2011 JINST 6 P09001 [arXiv:1106.5048] [INSPIRE].

[36] CMS collaboration, Search for narrow resonances in dilepton mass spectra in proton-proton collisions at $\sqrt{s}=13 \mathrm{TeV}$ and combination with $8 \mathrm{TeV}$ data, Phys. Lett. B 768 (2017) 57 [arXiv: 1609.05391] [INSPIRE].

[37] CMS collaboration, Performance of electron reconstruction and selection with the CMS detector in proton-proton collisions at $\sqrt{s}=8 \mathrm{TeV}, 2015$ JINST $10 \mathrm{P} 06005$ [arXiv: 1502.02701] [INSPIRE].

[38] CMS collaboration, Performance of CMS muon reconstruction in pp collision events at $\sqrt{s}=7$ TeV, 2012 JINST 7 P10002 [arXiv:1206.4071] [INSPIRE].

[39] CMS collaboration, Measurement of the inclusive $W$ and $Z$ production cross sections in $p p$ collisions at $\sqrt{s}=7 \mathrm{Te}$, JHEP 10 (2011) 132 [arXiv:1107.4789] [INSPIRE].

[40] CMS collaboration, Identification of heavy-flavour jets with the CMS detector in pp collisions at $13 \mathrm{TeV}, 2018$ JINST 13 P05011 [arXiv: 1712.07158] [INSPIRE].

[41] J. Alwall et al., The automated computation of tree-level and next-to-leading order differential cross sections and their matching to parton shower simulations, JHEP 07 (2014) 079 [arXiv: 1405.0301] [INSPIRE].

[42] NNPDF collaboration, R.D. Ball et al., Parton distributions from high-precision collider data, Eur. Phys. J. C 77 (2017) 663 [arXiv:1706.00428] [InSPIRE].

[43] S.G. Bondarenko and A.A. Sapronov, NLO EW and QCD proton-proton cross section calculations with mcsanc-v1.01, Comput. Phys. Commun. 184 (2013) 2343 [arXiv: 1301.3687] [INSPIRE].

[44] P. Nason, A new method for combining NLO QCD with shower Monte Carlo algorithms, JHEP 11 (2004) 040 [hep-ph/0409146] [INSPIRE].

[45] S. Frixione, P. Nason and C. Oleari, Matching NLO QCD computations with parton shower simulations: the POWHEG method, JHEP 11 (2007) 070 [arXiv:0709.2092] [INSPIRE].

[46] S. Alioli, P. Nason, C. Oleari and E. Re, A general framework for implementing NLO calculations in shower Monte Carlo programs: the POWHEG BOX, JHEP 06 (2010) 043 [arXiv:1002.2581] [INSPIRE].

[47] E. Re, Single-top Wt-channel production matched with parton showers using the POWHEG method, Eur. Phys. J. C 71 (2011) 1547 [arXiv: 1009.2450] [INSPIRE].

[48] M. Czakon, P. Fiedler and A. Mitov, Total top-quark pair-production cross section at hadron colliders through $O\left(\alpha_{S}^{4}\right)$, Phys. Rev. Lett. 110 (2013) 252004 [arXiv: 1303.6254] [INSPIRE].

[49] T. Gehrmann et al., $W^{+} W^{-}$production at hadron colliders in next to next to leading order QCD, Phys. Rev. Lett. 113 (2014) 212001 [arXiv:1408.5243] [InSPIRE].

[50] F. Cascioli et al., ZZ production at hadron colliders in NNLO QCD, Phys. Lett. B 735 (2014) 311 [arXiv:1405.2219] [INSPIRE]. 
[51] J.M. Campbell, R.K. Ellis and C. Williams, Vector boson pair production at the LHC, JHEP 07 (2011) 018 [arXiv: 1105.0020] [INSPIRE].

[52] J. Alwall et al., Comparative study of various algorithms for the merging of parton showers and matrix elements in hadronic collisions, Eur. Phys. J. C 53 (2008) 473 [arXiv:0706.2569] [INSPIRE].

[53] CMS collaboration, Energy calibration and resolution of the CMS electromagnetic calorimeter in pp collisions at $\sqrt{s}=7 \mathrm{TeV}, 2013$ JINST 8 P09009 [arXiv:1306.2016] [INSPIRE].

[54] J. Butterworth et al., PDF4LHC recommendations for LHC run II, J. Phys. G 43 (2016) 023001 [arXiv: 1510.03865] [INSPIRE].

[55] CMS collaboration, CMS luminosity measurements for the 2016 data taking period, CMS-LUM-17-001, CERN, Geneva, Switzerland, (2017).

[56] Particle Data Group collaboration, C. Patrignani et al., Review of particle physics, Chin. Phys. C 40 (2016) 100001 [inSPIRE]. 


\section{The CMS collaboration}

\section{Yerevan Physics Institute, Yerevan, Armenia}

A.M. Sirunyan, A. Tumasyan

\section{Institut für Hochenergiephysik, Wien, Austria}

W. Adam, F. Ambrogi, E. Asilar, T. Bergauer, J. Brandstetter, E. Brondolin, M. Dragicevic, J. Erö, A. Escalante Del Valle, M. Flechl, M. Friedl, R. Frühwirth ${ }^{1}$, V.M. Ghete, J. Grossmann, J. Hrubec, M. Jeitler ${ }^{1}$, A. König, N. Krammer, I. Krätschmer, D. Liko, T. Madlener, I. Mikulec, E. Pree, N. Rad, H. Rohringer, J. Schieck ${ }^{1}$, R. Schöfbeck, M. Spanring, D. Spitzbart, A. Taurok, W. Waltenberger, J. Wittmann, C.-E. Wulz ${ }^{1}$, M. Zarucki

\section{Institute for Nuclear Problems, Minsk, Belarus}

V. Chekhovsky, V. Mossolov, J. Suarez Gonzalez

\section{Universiteit Antwerpen, Antwerpen, Belgium}

E.A. De Wolf, D. Di Croce, X. Janssen, J. Lauwers, M. Pieters, M. Van De Klundert, H. Van Haevermaet, P. Van Mechelen, N. Van Remortel

\section{Vrije Universiteit Brussel, Brussel, Belgium}

S. Abu Zeid, F. Blekman, J. D'Hondt, I. De Bruyn, J. De Clercq, K. Deroover, G. Flouris, D. Lontkovskyi, S. Lowette, I. Marchesini, S. Moortgat, L. Moreels, Q. Python, K. Skovpen, S. Tavernier, W. Van Doninck, P. Van Mulders, I. Van Parijs

\section{Université Libre de Bruxelles, Bruxelles, Belgium}

D. Beghin, B. Bilin, H. Brun, B. Clerbaux, G. De Lentdecker, H. Delannoy, B. Dorney, G. Fasanella, L. Favart, R. Goldouzian, A. Grebenyuk, A.K. Kalsi, T. Lenzi, J. Luetic, T. Seva, E. Starling, C. Vander Velde, P. Vanlaer, D. Vannerom, R. Yonamine

\section{Ghent University, Ghent, Belgium}

T. Cornelis, D. Dobur, A. Fagot, M. Gul, I. Khvastunov², D. Poyraz, C. Roskas, D. Trocino, M. Tytgat, W. Verbeke, B. Vermassen, M. Vit, N. Zaganidis

\section{Université Catholique de Louvain, Louvain-la-Neuve, Belgium}

H. Bakhshiansohi, O. Bondu, S. Brochet, G. Bruno, C. Caputo, A. Caudron, P. David, S. De Visscher, C. Delaere, M. Delcourt, B. Francois, A. Giammanco, G. Krintiras, V. Lemaitre, A. Magitteri, A. Mertens, M. Musich, K. Piotrzkowski, L. Quertenmont, A. Saggio, M. Vidal Marono, S. Wertz, J. Zobec

\section{Centro Brasileiro de Pesquisas Fisicas, Rio de Janeiro, Brazil}

W.L. Aldá Júnior, F.L. Alves, G.A. Alves, L. Brito, G. Correia Silva, C. Hensel, A. Moraes, M.E. Pol, P. Rebello Teles

Universidade do Estado do Rio de Janeiro, Rio de Janeiro, Brazil

E. Belchior Batista Das Chagas, W. Carvalho, J. Chinellato ${ }^{3}$, E. Coelho, E.M. Da Costa, G.G. Da Silveira ${ }^{4}$, D. De Jesus Damiao, S. Fonseca De Souza, L.M. Huertas Guativa, H. Malbouisson, M. Medina Jaime ${ }^{5}$, M. Melo De Almeida, C. Mora Herrera, 
L. Mundim, H. Nogima, L.J. Sanchez Rosas, A. Santoro, A. Sznajder, M. Thiel, E.J. Tonelli Manganote $^{3}$, F. Torres Da Silva De Araujo, A. Vilela Pereira

Universidade Estadual Paulista $^{a}$, Universidade Federal do ABC ${ }^{b}$, São Paulo, Brazil

S. Ahuja ${ }^{a}$, C.A. Bernardes ${ }^{a}$, L. Calligaris ${ }^{a}$, T.R. Fernandez Perez Tomei ${ }^{a}$, E.M. Gregores $^{b}$, P.G. Mercadante ${ }^{b}$, S.F. Novaes ${ }^{a}$, Sandra S. Padula ${ }^{a}$, D. Romero $\operatorname{Abad}^{b}$, J.C. Ruiz Vargas ${ }^{a}$

Institute for Nuclear Research and Nuclear Energy, Bulgarian Academy of Sciences, Sofia, Bulgaria

A. Aleksandrov, R. Hadjiiska, P. Iaydjiev, A. Marinov, M. Misheva, M. Rodozov, M. Shopova, G. Sultanov

University of Sofia, Sofia, Bulgaria

A. Dimitrov, L. Litov, B. Pavlov, P. Petkov

Beihang University, Beijing, China

W. Fang ${ }^{6}$, X. Gao ${ }^{6}$, L. Yuan

Institute of High Energy Physics, Beijing, China

M. Ahmad, J.G. Bian, G.M. Chen, H.S. Chen, M. Chen, Y. Chen, C.H. Jiang, D. Leggat, H. Liao, Z. Liu, F. Romeo, S.M. Shaheen, A. Spiezia, J. Tao, C. Wang, Z. Wang, E. Yazgan, H. Zhang, J. Zhao

State Key Laboratory of Nuclear Physics and Technology, Peking University, Beijing, China

Y. Ban, G. Chen, J. Li, Q. Li, S. Liu, Y. Mao, S.J. Qian, D. Wang, Z. Xu

Tsinghua University, Beijing, China

Y. Wang

Universidad de Los Andes, Bogota, Colombia

C. Avila, A. Cabrera, C.A. Carrillo Montoya, L.F. Chaparro Sierra, C. Florez, C.F. González Hernández, M.A. Segura Delgado

University of Split, Faculty of Electrical Engineering, Mechanical Engineering and Naval Architecture, Split, Croatia

B. Courbon, N. Godinovic, D. Lelas, I. Puljak, P.M. Ribeiro Cipriano, T. Sculac

University of Split, Faculty of Science, Split, Croatia

Z. Antunovic, M. Kovac

Institute Rudjer Boskovic, Zagreb, Croatia

V. Brigljevic, D. Ferencek, K. Kadija, B. Mesic, A. Starodumov ${ }^{7}$, T. Susa

University of Cyprus, Nicosia, Cyprus

M.W. Ather, A. Attikis, G. Mavromanolakis, J. Mousa, C. Nicolaou, F. Ptochos, P.A. Razis, H. Rykaczewski

Charles University, Prague, Czech Republic

M. Finger ${ }^{8}$, M. Finger Jr. ${ }^{8}$ 
Universidad San Francisco de Quito, Quito, Ecuador

E. Carrera Jarrin

Academy of Scientific Research and Technology of the Arab Republic of Egypt, Egyptian Network of High Energy Physics, Cairo, Egypt

Y. Assran ${ }^{9,10}$, S. Elgammal ${ }^{10}$, A. Ellithi Kamel ${ }^{11}$

National Institute of Chemical Physics and Biophysics, Tallinn, Estonia

S. Bhowmik, R.K. Dewanjee, M. Kadastik, L. Perrini, M. Raidal, C. Veelken

Department of Physics, University of Helsinki, Helsinki, Finland

P. Eerola, H. Kirschenmann, J. Pekkanen, M. Voutilainen

Helsinki Institute of Physics, Helsinki, Finland

J. Havukainen, J.K. Heikkilä, T. Järvinen, V. Karimäki, R. Kinnunen, T. Lampén,

K. Lassila-Perini, S. Laurila, S. Lehti, T. Lindén, P. Luukka, T. Mäenpää, H. Siikonen,

E. Tuominen, J. Tuominiemi

Lappeenranta University of Technology, Lappeenranta, Finland

T. Tuuva

IRFU, CEA, Université Paris-Saclay, Gif-sur-Yvette, France

M. Besancon, F. Couderc, M. Dejardin, D. Denegri, J.L. Faure, F. Ferri, S. Ganjour, S. Ghosh, A. Givernaud, P. Gras, G. Hamel de Monchenault, P. Jarry, C. Leloup, E. Locci, M. Machet, J. Malcles, G. Negro, J. Rander, A. Rosowsky, M.Ö. Sahin, M. Titov

Laboratoire Leprince-Ringuet, Ecole polytechnique, CNRS/IN2P3, Université Paris-Saclay, Palaiseau, France

A. Abdulsalam ${ }^{12}$, C. Amendola, I. Antropov, S. Baffioni, F. Beaudette, P. Busson,

L. Cadamuro, C. Charlot, R. Granier de Cassagnac, M. Jo, I. Kucher, S. Lisniak,

A. Lobanov, J. Martin Blanco, M. Nguyen, C. Ochando, G. Ortona, P. Paganini, P. Pigard,

R. Salerno, J.B. Sauvan, Y. Sirois, A.G. Stahl Leiton, Y. Yilmaz, A. Zabi, A. Zghiche

Université de Strasbourg, CNRS, IPHC UMR 7178, F-67000 Strasbourg, France

J.-L. Agram ${ }^{13}$, J. Andrea, D. Bloch, J.-M. Brom, M. Buttignol, E.C. Chabert, C. Collard, E. Conte ${ }^{13}$, X. Coubez, F. Drouhin ${ }^{13}$, J.-C. Fontaine ${ }^{13}$, D. Gelé, U. Goerlach, M. Jansová, P. Juillot, A.-C. Le Bihan, N. Tonon, P. Van Hove

Centre de Calcul de l'Institut National de Physique Nucleaire et de Physique des Particules, CNRS/IN2P3, Villeurbanne, France

S. Gadrat

Université de Lyon, Université Claude Bernard Lyon 1, CNRS-IN2P3, Institut de Physique Nucléaire de Lyon, Villeurbanne, France

S. Beauceron, C. Bernet, G. Boudoul, N. Chanon, R. Chierici, D. Contardo, P. Depasse, H. El Mamouni, J. Fay, L. Finco, S. Gascon, M. Gouzevitch, G. Grenier, B. Ille, F. Lagarde, I.B. Laktineh, H. Lattaud, M. Lethuillier, L. Mirabito, A.L. Pequegnot, S. Perries, A. Popov ${ }^{14}$, V. Sordini, M. Vander Donckt, S. Viret, S. Zhang 
Georgian Technical University, Tbilisi, Georgia

T. Toriashvili ${ }^{15}$

Tbilisi State University, Tbilisi, Georgia

Z. Tsamalaidze ${ }^{8}$

RWTH Aachen University, I. Physikalisches Institut, Aachen, Germany

C. Autermann, L. Feld, M.K. Kiesel, K. Klein, M. Lipinski, M. Preuten, C. Schomakers, J. Schulz, M. Teroerde, B. Wittmer, V. Zhukov ${ }^{14}$

RWTH Aachen University, III. Physikalisches Institut A, Aachen, Germany

A. Albert, F.F. Bispinck, D. Duchardt, M. Endres, M. Erdmann, S. Erdweg, T. Esch, R. Fischer, A. Güth, T. Hebbeker, C. Heidemann, K. Hoepfner, S. Knutzen, M. Materok, M. Merschmeyer, A. Meyer, P. Millet, S. Mukherjee, T. Pook, M. Radziej, H. Reithler, M. Rieger, F. Scheuch, D. Teyssier, S. Thüer, S. Wiedenbeck

RWTH Aachen University, III. Physikalisches Institut B, Aachen, Germany

G. Flügge, B. Kargoll, T. Kress, A. Künsken, T. Müller, A. Nehrkorn, A. Nowack, C. Pistone, O. Pooth, A. Stahl ${ }^{16}$

\section{Deutsches Elektronen-Synchrotron, Hamburg, Germany}

M. Aldaya Martin, T. Arndt, C. Asawatangtrakuldee, K. Beernaert, O. Behnke, U. Behrens, A. Bermúdez Martínez, A.A. Bin Anuar, K. Borras ${ }^{17}$, V. Botta, A. Campbell, P. Connor, C. Contreras-Campana, F. Costanza, V. Danilov, A. De Wit, C. Diez Pardos, D. Domínguez Damiani, G. Eckerlin, D. Eckstein, T. Eichhorn, E. Eren, E. Gallo ${ }^{18}$, J. Garay Garcia, A. Geiser, J.M. Grados Luyando, A. Grohsjean, P. Gunnellini, M. Guthoff, A. Harb, J. Hauk, M. Hempel ${ }^{19}$, H. Jung, M. Kasemann, J. Keaveney, C. Kleinwort, J. Knolle, I. Korol, D. Krücker, W. Lange, A. Lelek, T. Lenz, K. Lipka, W. Lohmann ${ }^{19}$, R. Mankel, I.-A. Melzer-Pellmann, A.B. Meyer, M. Meyer, M. Missiroli, G. Mittag, J. Mnich, A. Mussgiller, D. Pitzl, A. Raspereza, M. Savitskyi, P. Saxena, R. Shevchenko, N. Stefaniuk, H. Tholen, G.P. Van Onsem, R. Walsh, Y. Wen, K. Wichmann, C. Wissing, O. Zenaiev

\section{University of Hamburg, Hamburg, Germany}

R. Aggleton, S. Bein, V. Blobel, M. Centis Vignali, T. Dreyer, E. Garutti, D. Gonzalez, J. Haller, A. Hinzmann, M. Hoffmann, A. Karavdina, G. Kasieczka, R. Klanner, R. Kogler, N. Kovalchuk, S. Kurz, D. Marconi, J. Multhaup, M. Niedziela, D. Nowatschin, T. Peiffer, A. Perieanu, A. Reimers, C. Scharf, P. Schleper, A. Schmidt, S. Schumann, J. Schwandt, J. Sonneveld, H. Stadie, G. Steinbrück, F.M. Stober, M. Stöver, D. Troendle, E. Usai, A. Vanhoefer, B. Vormwald

\section{Institut für Experimentelle Teilchenphysik, Karlsruhe, Germany}

M. Akbiyik, C. Barth, M. Baselga, S. Baur, E. Butz, R. Caspart, T. Chwalek, F. Colombo, W. De Boer, A. Dierlamm, N. Faltermann, B. Freund, R. Friese, M. Giffels, M.A. Harrendorf, F. Hartmann ${ }^{16}$, S.M. Heindl, U. Husemann, F. Kassel ${ }^{16}$, S. Kudella, H. Mildner, M.U. Mozer, Th. Müller, M. Plagge, G. Quast, K. Rabbertz, M. Schröder, I. Shvetsov, 
G. Sieber, H.J. Simonis, R. Ulrich, S. Wayand, M. Weber, T. Weiler, S. Williamson, C. Wöhrmann, R. Wolf

Institute of Nuclear and Particle Physics (INPP), NCSR Demokritos, Aghia Paraskevi, Greece

G. Anagnostou, G. Daskalakis, T. Geralis, A. Kyriakis, D. Loukas, I. Topsis-Giotis

National and Kapodistrian University of Athens, Athens, Greece

G. Karathanasis, S. Kesisoglou, A. Panagiotou, N. Saoulidou, E. Tziaferi

National Technical University of Athens, Athens, Greece

K. Kousouris, I. Papakrivopoulos

University of Ioánnina, Ioánnina, Greece

I. Evangelou, C. Foudas, P. Gianneios, P. Katsoulis, P. Kokkas, S. Mallios, N. Manthos,

I. Papadopoulos, E. Paradas, J. Strologas, F.A. Triantis, D. Tsitsonis

MTA-ELTE Lendület CMS Particle and Nuclear Physics Group, Eötvös Loránd University, Budapest, Hungary

M. Csanad, N. Filipovic, G. Pasztor, O. Surányi, G.I. Veres ${ }^{20}$

Wigner Research Centre for Physics, Budapest, Hungary

G. Bencze, C. Hajdu, D. Horvath ${ }^{21}$, Á. Hunyadi, F. Sikler, V. Veszpremi, G. Vesztergombi ${ }^{20}$, T.Á. Vámi

Institute of Nuclear Research ATOMKI, Debrecen, Hungary

N. Beni, S. Czellar, J. Karancsi ${ }^{22}$, A. Makovec, J. Molnar, Z. Szillasi

Institute of Physics, University of Debrecen, Debrecen, Hungary

M. Bartók ${ }^{20}$, P. Raics, Z.L. Trocsanyi, B. Ujvari

Indian Institute of Science (IISc), Bangalore, India

S. Choudhury, J.R. Komaragiri

National Institute of Science Education and Research, Bhubaneswar, India

S. Bahinipatii ${ }^{23}$, P. Mal, K. Mandal, A. Nayak ${ }^{24}$, D.K. Sahoo ${ }^{23}$, N. Sahoo, S.K. Swain

Panjab University, Chandigarh, India

S. Bansal, S.B. Beri, V. Bhatnagar, S. Chauhan, R. Chawla, N. Dhingra, R. Gupta, A. Kaur, M. Kaur, S. Kaur, R. Kumar, P. Kumari, M. Lohan, A. Mehta, S. Sharma, J.B. Singh, G. Walia

University of Delhi, Delhi, India

Ashok Kumar, Aashaq Shah, A. Bhardwaj, B.C. Choudhary, R.B. Garg, S. Keshri, A. Kumar, S. Malhotra, M. Naimuddin, K. Ranjan, R. Sharma

Saha Institute of Nuclear Physics, HBNI, Kolkata, India

R. Bhardwaj ${ }^{25}$, R. Bhattacharya, S. Bhattacharya, U. Bhawandeep ${ }^{25}$, D. Bhowmik, S. Dey, S. Dutt ${ }^{25}$, S. Dutta, S. Ghosh, N. Majumdar, K. Mondal, S. Mukhopadhyay, S. Nandan, A. Purohit, P.K. Rout, A. Roy, S. Roy Chowdhury, S. Sarkar, M. Sharan, B. Singh, S. Thakur ${ }^{25}$ 
Indian Institute of Technology Madras, Madras, India

P.K. Behera

Bhabha Atomic Research Centre, Mumbai, India

R. Chudasama, D. Dutta, V. Jha, V. Kumar, A.K. Mohanty ${ }^{16}$, P.K. Netrakanti, L.M. Pant, P. Shukla, A. Topkar

Tata Institute of Fundamental Research-A, Mumbai, India

T. Aziz, S. Dugad, B. Mahakud, S. Mitra, G.B. Mohanty, N. Sur, B. Sutar

Tata Institute of Fundamental Research-B, Mumbai, India

S. Banerjee, S. Bhattacharya, S. Chatterjee, P. Das, M. Guchait, Sa. Jain, S. Kumar, M. Maity ${ }^{26}$, G. Majumder, K. Mazumdar, T. Sarkar ${ }^{26}$, N. Wickramage ${ }^{27}$

Indian Institute of Science Education and Research (IISER), Pune, India

S. Chauhan, S. Dube, V. Hegde, A. Kapoor, K. Kothekar, S. Pandey, A. Rane, S. Sharma

Institute for Research in Fundamental Sciences (IPM), Tehran, Iran

S. Chenarani ${ }^{28}$, E. Eskandari Tadavani, S.M. Etesami ${ }^{28}$, M. Khakzad, M. Mohammadi Najafabadi, M. Naseri, S. Paktinat Mehdiabadi ${ }^{29}$, F. Rezaei Hosseinabadi, B. Safarzadeh ${ }^{30}$, M. Zeinali

University College Dublin, Dublin, Ireland

M. Felcini, M. Grunewald

INFN Sezione di Bari ${ }^{a}$, Università di Bari ${ }^{b}$, Politecnico di Bari ${ }^{c}$, Bari, Italy M. Abbrescia ${ }^{a, b}$, C. Calabria ${ }^{a, b}$, A. Colaleo ${ }^{a}$, D. Creanza ${ }^{a, c}$, L. Cristella ${ }^{a, b}$, N. De Filippis $^{a, c}$, M. De Palma ${ }^{a, b}$, A. Di Florio ${ }^{a, b}$, F. Errico ${ }^{a, b}$, L. Fiore ${ }^{a}$, A. Gelmi ${ }^{a, b}$,

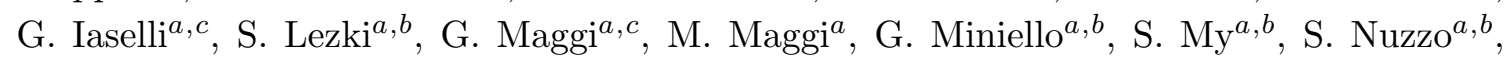
A. Pompili ${ }^{a, b}$, G. Pugliese ${ }^{a, c}$, R. Radogna ${ }^{a}$, A. Ranieri ${ }^{a}$, G. Selvaggi ${ }^{a, b}$, A. Sharma ${ }^{a}$, L. Silvestris ${ }^{a, 16}$, R. Venditti ${ }^{a}$, P. Verwilligen ${ }^{a}$

INFN Sezione di Bologna ${ }^{a}$, Università di Bologna ${ }^{b}$, Bologna, Italy

G. Abbiendi ${ }^{a}$, C. Battilana ${ }^{a, b}$, D. Bonacorsi ${ }^{a}, b$, L. Borgonovi $^{a, b}$, S. Braibant-Giacomelli ${ }^{a, b}$, R. Campanini ${ }^{a, b}$, P. Capiluppi ${ }^{a, b}$, A. Castro ${ }^{a, b}$, F.R. Cavallo ${ }^{a}$, S.S. Chhibra ${ }^{a, b}$, G. Codispoti ${ }^{a, b}$, M. Cuffiani ${ }^{a, b}$, G.M. Dallavalle ${ }^{a}$, F. Fabbri $^{a}$, A. Fanfani ${ }^{a, b}$, D. Fasanella ${ }^{a, b}$, P. Giacomelli ${ }^{a}$, C. Grandi ${ }^{a}$, L. Guiducci ${ }^{a}, b$, S. Marcellini ${ }^{a}$, G. Masetti ${ }^{a}$, A. Montanari ${ }^{a}$, F.L. Navarria ${ }^{a, b}$, F. Odorici ${ }^{a}$, A. Perrotta ${ }^{a}$, A.M. Rossi ${ }^{a, b}$, T. Rovelli ${ }^{a, b}$, G.P. Siroli ${ }^{a, b}$, N. $\operatorname{Tosi}^{a}$

INFN Sezione di Catania ${ }^{a}$, Università di Catania ${ }^{b}$, Catania, Italy

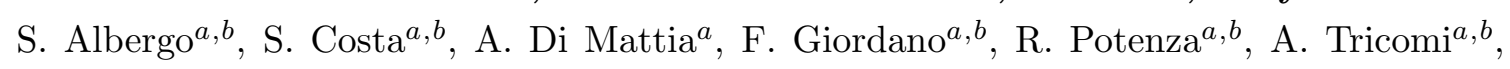
C. Tuve ${ }^{a, b}$

INFN Sezione di Firenze ${ }^{a}$, Università di Firenze ${ }^{b}$, Firenze, Italy

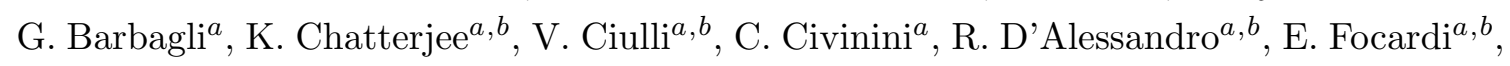
G. Latino, P. Lenzi ${ }^{a, b}$, M. Meschini ${ }^{a}$, S. Paoletti ${ }^{a}$, L. Russo $^{a}, 31$, G. Sguazzoni $^{a}$, D. Strom ${ }^{a}$, L. Viliani ${ }^{a}$ 
INFN Laboratori Nazionali di Frascati, Frascati, Italy

L. Benussi, S. Bianco, F. Fabbri, D. Piccolo, F. Primavera ${ }^{16}$

INFN Sezione di Genova ${ }^{a}$, Università di Genova ${ }^{b}$, Genova, Italy

V. Calvelli ${ }^{a}, b$, F. Ferro ${ }^{a}$, F. Ravera ${ }^{a}, b$ E. Robutti ${ }^{a}$, S. Tosi ${ }^{a, b}$

INFN Sezione di Milano-Bicocca ${ }^{a}$, Università di Milano-Bicocca ${ }^{b}$, Milano, Italy

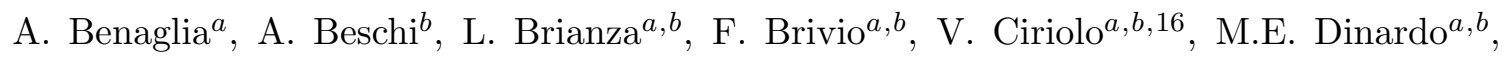
S. Fiorendi ${ }^{a, b}$, S. Gennai ${ }^{a}$, A. Ghezzi ${ }^{a, b}$, P. Govoni ${ }^{a, b}$, M. Malberti ${ }^{a, b}$, S. Malvezzi ${ }^{a}$, R.A. Manzoni ${ }^{a}, b$, D. Menasce ${ }^{a}$, L. Moroni ${ }^{a}$, M. Paganoni ${ }^{a}, b$, K. Pauwels ${ }^{a}, b$, D. Pedrini ${ }^{a}$, S. Pigazzini ${ }^{a, b, 32}$, S. Ragazzi ${ }^{a, b}$, T. Tabarelli de Fatis ${ }^{a, b}$

INFN Sezione di Napoli ${ }^{a}$, Università di Napoli 'Federico II' ${ }^{b}$, Napoli, Italy, Università della Basilicata ${ }^{c}$, Potenza, Italy, Università G. Marconi ${ }^{d}$, Roma, Italy

S. Buontempo ${ }^{a}$, N. Cavallo ${ }^{a, c}$, S. Di Guida ${ }^{a, d, 16}$, F. Fabozzi $^{a, c}$, F. Fienga $^{a, b}$, G. Galati $^{a, b}$, A.O.M. Iorio ${ }^{a, b}$, W.A. Khan ${ }^{a}$, L. Lista ${ }^{a}$, S. Meola ${ }^{a, d, 16}$, P. Paolucci ${ }^{a, 16}$, C. Sciacca ${ }^{a, b}$, F. Thyssen ${ }^{a}$, E. Voevodina ${ }^{a, b}$

INFN Sezione di Padova ${ }^{a}$, Università di Padova ${ }^{b}$, Padova, Italy, Università di Trento ${ }^{c}$, Trento, Italy

P. Azzi ${ }^{a}$, N. Bacchetta ${ }^{a}$, L. Benato ${ }^{a, b}$, D. Bisello ${ }^{a, b}$, A. Boletti ${ }^{a, b}$, R. Carlin ${ }^{a, b}$, A. Carvalho Antunes De Oliveira ${ }^{a, b}$, P. Checchia ${ }^{a}$, P. De Castro Manzano ${ }^{a}$, T. Dorigo ${ }^{a}$,

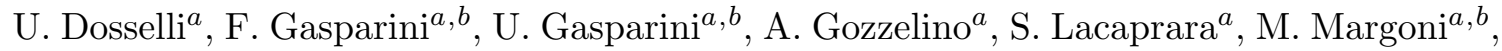
A.T. Meneguzzo ${ }^{a, b}$, N. Pozzobon ${ }^{a, b}$, P. Ronchese ${ }^{a, b}$, R. Rossin $^{a, b}$, F. Simonetto ${ }^{a, b}$, A. Tiko, E. Torassa ${ }^{a}$, M. Zanetti ${ }^{a, b}$, P. Zotto ${ }^{a, b}$, G. Zumerle ${ }^{a, b}$

INFN Sezione di Pavia ${ }^{a}$, Università di Pavia ${ }^{b}$, Pavia, Italy

A. Braghieri ${ }^{a}$, A. Magnani ${ }^{a}$, P. Montagna ${ }^{a, b}$, S.P. Ratti ${ }^{a, b}$, V. $\operatorname{Re}^{a}$, M. Ressegotti $^{a, b}$, C. Riccardi ${ }^{a, b}$, P. Salvini ${ }^{a}$, I. Vai ${ }^{a}, b$, P. Vitulo ${ }^{a, b}$

INFN Sezione di Perugia ${ }^{a}$, Università di Perugia ${ }^{b}$, Perugia, Italy

L. Alunni Solestizi ${ }^{a, b}$, M. Biasini ${ }^{a, b}$, G.M. Bilei ${ }^{a}$, C. Cecchi $^{a, b}$, D. Ciangottini ${ }^{a, b}$, L. Fanò $^{a, b}$,

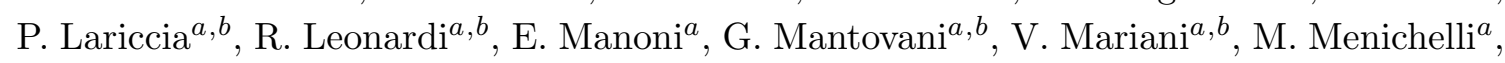
A. $\operatorname{Rossi}^{a, b}$, A. Santocchia ${ }^{a, b}$, D. Spiga ${ }^{a}$

INFN Sezione di Pisa ${ }^{a}$, Università di Pisa ${ }^{b}$, Scuola Normale Superiore di Pisa ${ }^{c}$, Pisa, Italy

K. Androsov ${ }^{a}$, P. Azzurri ${ }^{a}, 16$, G. Bagliesi ${ }^{a}$, L. Bianchini ${ }^{a}$, T. Boccali ${ }^{a}$, L. Borrello,

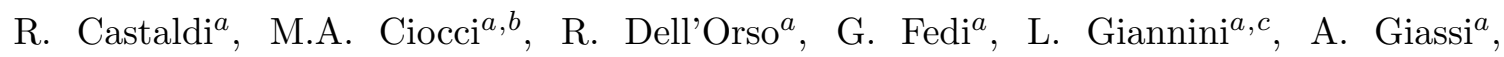
M.T. Grippo ${ }^{a, 31}$, F. Ligabue ${ }^{a, c}$, T. Lomtadze ${ }^{a}$, E. Manca ${ }^{a, c}$, G. Mandorlia ${ }^{a, c}$,

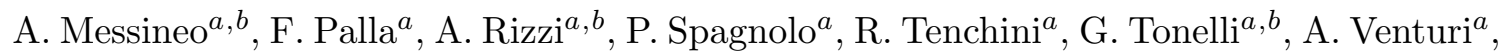
P.G. Verdini ${ }^{a}$

INFN Sezione di Roma ${ }^{a}$, Sapienza Università di Roma ${ }^{b}$, Rome, Italy

L. Barone ${ }^{a, b}$, F. Cavallari ${ }^{a}$, M. Cipriani ${ }^{a, b}$, N. Daci ${ }^{a}$, D. Del Re Re $^{a, b}$ E. Di Marco ${ }^{a, b}$, M. Diemoz ${ }^{a}$, S. Gelli ${ }^{a, b}$, E. Longo ${ }^{a, b}$, F. Margaroli ${ }^{a, b}$, B. Marzocchi ${ }^{a} b$, P. Meridiani ${ }^{a}$, 


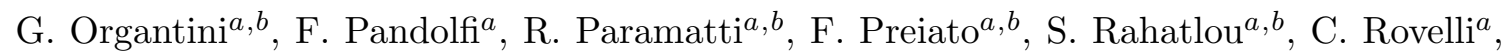
F. Santanastasio ${ }^{a, b}$

INFN Sezione di Torino ${ }^{a}$, Università di Torino ${ }^{b}$, Torino, Italy, Università del Piemonte Orientale ${ }^{c}$, Novara, Italy

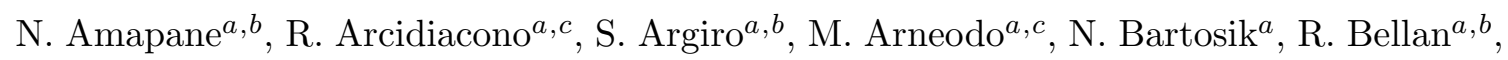

C. Biino ${ }^{a}$, N. Cartiglia ${ }^{a}$, R. Castello ${ }^{a, b}$, F. Cenna ${ }^{a, b}$, M. Costa $^{a, b}$, R. Covarelli ${ }^{a, b}$,

A. Degano ${ }^{a, b}$, N. $\operatorname{Demaria}^{a}$, B. Kiani ${ }^{a, b}$, C. Mariotti ${ }^{a}$, S. Maselli ${ }^{a}$, E. Migliore ${ }^{a, b}$,

V. Monaco ${ }^{a, b}$, E. Monteil ${ }^{a, b}$, M. Monteno ${ }^{a}$, M.M. Obertino ${ }^{a, b}$, L. Pacher $^{a, b}$, N. Pastrone ${ }^{a}$,

M. Pelliccioni ${ }^{a}$, G.L. Pinna Angioni ${ }^{a, b}$, A. Romero ${ }^{a, b}$, M. Ruspa ${ }^{a, c}$, R. Sacchi $^{a, b}$,

K. Shchelina ${ }^{a, b}$, V. Sola ${ }^{a}$, A. Solano ${ }^{a, b}$, A. Staiano ${ }^{a}$

INFN Sezione di Trieste ${ }^{a}$, Università di Trieste ${ }^{b}$, Trieste, Italy

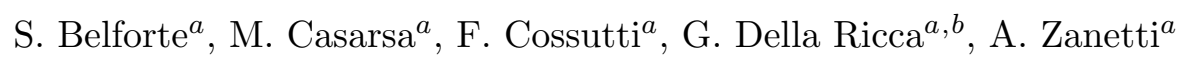

Kyungpook National University

D.H. Kim, G.N. Kim, M.S. Kim, J. Lee, S. Lee, S.W. Lee, C.S. Moon, Y.D. Oh, S. Sekmen, D.C. Son, Y.C. Yang

Chonnam National University, Institute for Universe and Elementary Particles, Kwangju, Korea

H. Kim, D.H. Moon, G. Oh

Hanyang University, Seoul, Korea

J.A. Brochero Cifuentes, J. Goh, T.J. Kim

Korea University, Seoul, Korea

S. Cho, S. Choi, Y. Go, D. Gyun, S. Ha, B. Hong, Y. Jo, Y. Kim, K. Lee, K.S. Lee, S. Lee, J. Lim, S.K. Park, Y. Roh

Seoul National University, Seoul, Korea

J. Almond, J. Kim, J.S. Kim, H. Lee, K. Lee, K. Nam, S.B. Oh, B.C. Radburn-Smith, S.h. Seo, U.K. Yang, H.D. Yoo, G.B. Yu

University of Seoul, Seoul, Korea

H. Kim, J.H. Kim, J.S.H. Lee, I.C. Park

Sungkyunkwan University, Suwon, Korea

Y. Choi, C. Hwang, J. Lee, I. Yu

Vilnius University, Vilnius, Lithuania

V. Dudenas, A. Juodagalvis, J. Vaitkus

National Centre for Particle Physics, Universiti Malaya, Kuala Lumpur, Malaysia

I. Ahmed, Z.A. Ibrahim, M.A.B. Md $\mathrm{Ali}^{33}$, F. Mohamad Idris ${ }^{34}$, W.A.T. Wan Abdullah, M.N. Yusli, Z. Zolkapli 
Centro de Investigacion y de Estudios Avanzados del IPN, Mexico City, Mexico Reyes-Almanza, R, Ramirez-Sanchez, G., Duran-Osuna, M. C., H. Castilla-Valdez, E. De La Cruz-Burelo, I. Heredia-De La Cruz ${ }^{35}$, Rabadan-Trejo, R. I., R. Lopez-Fernandez, J. Mejia Guisao, A. Sanchez-Hernandez

Universidad Iberoamericana, Mexico City, Mexico

S. Carrillo Moreno, C. Oropeza Barrera, F. Vazquez Valencia

Benemerita Universidad Autonoma de Puebla, Puebla, Mexico

J. Eysermans, I. Pedraza, H.A. Salazar Ibarguen, C. Uribe Estrada

Universidad Autónoma de San Luis Potosí, San Luis Potosí, Mexico

A. Morelos Pineda

University of Auckland, Auckland, New Zealand

D. Krofcheck

University of Canterbury, Christchurch, New Zealand

S. Bheesette, P.H. Butler

National Centre for Physics, Quaid-I-Azam University, Islamabad, Pakistan

A. Ahmad, M. Ahmad, Q. Hassan, H.R. Hoorani, A. Saddique, M.A. Shah, M. Shoaib, M. Waqas

National Centre for Nuclear Research, Swierk, Poland

H. Bialkowska, M. Bluj, B. Boimska, T. Frueboes, M. Górski, M. Kazana, K. Nawrocki, M. Szleper, P. Traczyk, P. Zalewski

Institute of Experimental Physics, Faculty of Physics, University of Warsaw, Warsaw, Poland

K. Bunkowski, A. Byszuk ${ }^{36}$, K. Doroba, A. Kalinowski, M. Konecki, J. Krolikowski, M. Misiura, M. Olszewski, A. Pyskir, M. Walczak

Laboratório de Instrumentação e Física Experimental de Partículas, Lisboa, Portugal

P. Bargassa, C. Beirão Da Cruz E Silva, A. Di Francesco, P. Faccioli, B. Galinhas, M. Gallinaro, J. Hollar, N. Leonardo, L. Lloret Iglesias, M.V. Nemallapudi, J. Seixas, G. Strong, O. Toldaiev, D. Vadruccio, J. Varela

Joint Institute for Nuclear Research, Dubna, Russia

S. Afanasiev, P. Bunin, M. Gavrilenko, I. Golutvin, I. Gorbunov, A. Kamenev, V. Karjavin, A. Lanev, A. Malakhov, V. Matveev ${ }^{37,38}$, P. Moisenz, V. Palichik, V. Perelygin, S. Shmatov, S. Shulha, N. Skatchkov, V. Smirnov, N. Voytishin, A. Zarubin

Petersburg Nuclear Physics Institute, Gatchina (St. Petersburg), Russia

Y. Ivanov, V. Kim ${ }^{39}$, E. Kuznetsova ${ }^{40}$, P. Levchenko, V. Murzin, V. Oreshkin, I. Smirnov, D. Sosnov, V. Sulimov, L. Uvarov, S. Vavilov, A. Vorobyev 
Institute for Nuclear Research, Moscow, Russia

Yu. Andreev, A. Dermenev, S. Gninenko, N. Golubev, A. Karneyeu, M. Kirsanov, N. Krasnikov, A. Pashenkov, D. Tlisov, A. Toropin

Institute for Theoretical and Experimental Physics, Moscow, Russia

V. Epshteyn, V. Gavrilov, N. Lychkovskaya, V. Popov, I. Pozdnyakov, G. Safronov, A. Spiridonov, A. Stepennov, V. Stolin, M. Toms, E. Vlasov, A. Zhokin

Moscow Institute of Physics and Technology, Moscow, Russia

T. Aushev, A. Bylinkin ${ }^{38}$

National Research Nuclear University 'Moscow Engineering Physics Institute' (MEPhI), Moscow, Russia

M. Chadeeva ${ }^{41}$, P. Parygin, D. Philippov, S. Polikarpov, E. Popova, V. Rusinov

P.N. Lebedev Physical Institute, Moscow, Russia

V. Andreev, M. Azarkin ${ }^{38}$, I. Dremin ${ }^{38}$, M. Kirakosyan ${ }^{38}$, S.V. Rusakov, A. Terkulov

Skobeltsyn Institute of Nuclear Physics, Lomonosov Moscow State University, Moscow, Russia

A. Baskakov, A. Belyaev, E. Boos, M. Dubinin ${ }^{42}$, L. Dudko, A. Ershov, A. Gribushin, V. Klyukhin, O. Kodolova, I. Lokhtin, I. Miagkov, S. Obraztsov, S. Petrushanko, V. Savrin, A. Snigirev

Novosibirsk State University (NSU), Novosibirsk, Russia V. Blinov ${ }^{43}$, D. Shtol ${ }^{43}$, Y. Skovpen ${ }^{43}$

State Research Center of Russian Federation, Institute for High Energy Physics of NRC \&quot;Kurchatov Institute\&quot;, Protvino, Russia

I. Azhgirey, I. Bayshev, S. Bitioukov, D. Elumakhov, A. Godizov, V. Kachanov, A. Kalinin, D. Konstantinov, P. Mandrik, V. Petrov, R. Ryutin, A. Sobol, S. Troshin, N. Tyurin, A. Uzunian, A. Volkov

National Research Tomsk Polytechnic University, Tomsk, Russia

A. Babaev

University of Belgrade, Faculty of Physics and Vinca Institute of Nuclear Sciences, Belgrade, Serbia

P. Adzic ${ }^{44}$, P. Cirkovic, D. Devetak, M. Dordevic, J. Milosevic

Centro de Investigaciones Energéticas Medioambientales y Tecnológicas (CIEMAT), Madrid, Spain

J. Alcaraz Maestre, I. Bachiller, M. Barrio Luna, M. Cerrada, N. Colino, B. De La Cruz, A. Delgado Peris, C. Fernandez Bedoya, J.P. Fernández Ramos, J. Flix, M.C. Fouz, O. Gonzalez Lopez, S. Goy Lopez, J.M. Hernandez, M.I. Josa, D. Moran, A. Pérez-Calero Yzquierdo, J. Puerta Pelayo, I. Redondo, L. Romero, M.S. Soares, A. Triossi, A. Álvarez Fernández 
Universidad Autónoma de Madrid, Madrid, Spain

C. Albajar, J.F. de Trocóniz

\section{Universidad de Oviedo, Oviedo, Spain}

J. Cuevas, C. Erice, J. Fernandez Menendez, S. Folgueras, I. Gonzalez Caballero, J.R. González Fernández, E. Palencia Cortezon, S. Sanchez Cruz, P. Vischia, J.M. Vizan Garcia

Instituto de Física de Cantabria (IFCA), CSIC-Universidad de Cantabria, Santander, Spain

I.J. Cabrillo, A. Calderon, B. Chazin Quero, J. Duarte Campderros, M. Fernandez, P.J. Fernández Manteca, J. Garcia-Ferrero, A. García Alonso, G. Gomez, A. Lopez Virto, J. Marco, C. Martinez Rivero, P. Martinez Ruiz del Arbol, F. Matorras, J. Piedra Gomez, C. Prieels, T. Rodrigo, A. Ruiz-Jimeno, L. Scodellaro, N. Trevisani, I. Vila, R. Vilar Cortabitarte

\section{CERN, European Organization for Nuclear Research, Geneva, Switzerland}

D. Abbaneo, B. Akgun, E. Auffray, P. Baillon, A.H. Ball, D. Barney, J. Bendavid, M. Bianco, A. Bocci, C. Botta, T. Camporesi, M. Cepeda, G. Cerminara, E. Chapon, Y. Chen, D. d'Enterria, A. Dabrowski, V. Daponte, A. David, M. De Gruttola, A. De Roeck, N. Deelen, M. Dobson, T. du Pree, M. Dünser, N. Dupont, A. Elliott-Peisert, P. Everaerts, F. Fallavollita ${ }^{45}$, G. Franzoni, J. Fulcher, W. Funk, D. Gigi, A. Gilbert, K. Gill, F. Glege, D. Gulhan, J. Hegeman, V. Innocente, A. Jafari, P. Janot, O. Karacheban ${ }^{19}$, J. Kieseler, V. Knünz, A. Kornmayer, M. Krammer ${ }^{1}$, C. Lange, P. Lecoq, C. Lourenço, M.T. Lucchini, L. Malgeri, M. Mannelli, A. Martelli, F. Meijers, J.A. Merlin, S. Mersi, E. Meschi, P. Milenovic ${ }^{46}$, F. Moortgat, M. Mulders, H. Neugebauer, J. Ngadiuba, S. Orfanelli, L. Orsini, F. Pantaleo ${ }^{16}$, L. Pape, E. Perez, M. Peruzzi, A. Petrilli, G. Petrucciani, A. Pfeiffer, M. Pierini, F.M. Pitters, D. Rabady, A. Racz, T. Reis, G. Rolandi ${ }^{47}$, M. Rovere, H. Sakulin, C. Schäfer, C. Schwick, M. Seidel, M. Selvaggi, A. Sharma, P. Silva, P. Sphicas ${ }^{48}$, A. Stakia, J. Steggemann, M. Stoye, M. Tosi, D. Treille, A. Tsirou, V. Veckalns ${ }^{49}$, M. Verweij, W.D. Zeuner

\section{Paul Scherrer Institut, Villigen, Switzerland}

W. Bertl ${ }^{\dagger}$, L. Caminada ${ }^{50}$, K. Deiters, W. Erdmann, R. Horisberger, Q. Ingram, H.C. Kaestli, D. Kotlinski, U. Langenegger, T. Rohe, S.A. Wiederkehr

ETH Zurich - Institute for Particle Physics and Astrophysics (IPA), Zurich, Switzerland

M. Backhaus, L. Bäni, P. Berger, B. Casal, N. Chernyavskaya, G. Dissertori, M. Dittmar, M. Donegà, C. Dorfer, C. Grab, C. Heidegger, D. Hits, J. Hoss, T. Klijnsma, W. Lustermann, M. Marionneau, M.T. Meinhard, D. Meister, F. Micheli, P. Musella, F. NessiTedaldi, J. Pata, F. Pauss, G. Perrin, L. Perrozzi, M. Quittnat, M. Reichmann, D. Ruini, D.A. Sanz Becerra, M. Schönenberger, L. Shchutska, V.R. Tavolaro, K. Theofilatos, M.L. Vesterbacka Olsson, R. Wallny, D.H. Zhu 
Universität Zürich, Zurich, Switzerland

T.K. Aarrestad, C. Amsler ${ }^{51}$, D. Brzhechko, M.F. Canelli, A. De Cosa, R. Del Burgo, S. Donato, C. Galloni, T. Hreus, B. Kilminster, I. Neutelings, D. Pinna, G. Rauco, P. Robmann, D. Salerno, K. Schweiger, C. Seitz, Y. Takahashi, A. Zucchetta

National Central University, Chung-Li, Taiwan

V. Candelise, Y.H. Chang, K.y. Cheng, T.H. Doan, Sh. Jain, R. Khurana, C.M. Kuo, W. Lin, A. Pozdnyakov, S.S. Yu

National Taiwan University (NTU), Taipei, Taiwan

Arun Kumar, P. Chang, Y. Chao, K.F. Chen, P.H. Chen, F. Fiori, W.-S. Hou, Y. Hsiung, Y.F. Liu, R.-S. Lu, E. Paganis, A. Psallidas, A. Steen, J.f. Tsai

Chulalongkorn University, Faculty of Science, Department of Physics, Bangkok, Thailand

B. Asavapibhop, K. Kovitanggoon, G. Singh, N. Srimanobhas

Çukurova University, Physics Department, Science and Art Faculty, Adana, Turkey

M.N. Bakirci ${ }^{52}$, A. Bat, F. Boran, S. Damarseckin, Z.S. Demiroglu, C. Dozen, E. Eskut, S. Girgis, G. Gokbulut, Y. Guler, I. Hos ${ }^{53}$, E.E. Kangal ${ }^{54}$, O. Kara, U. Kiminsu, M. Oglakci, G. Onengut, K. Ozdemir ${ }^{55}$, S. Ozturk ${ }^{52}$, A. Polatoz, D. Sunar Cerci ${ }^{56}$, U.G. Tok, S. Turkcapar, I.S. Zorbakir, C. Zorbilmez

Middle East Technical University, Physics Department, Ankara, Turkey

G. Karapinar ${ }^{57}$, K. Ocalan ${ }^{58}$, M. Yalvac, M. Zeyrek

Bogazici University, Istanbul, Turkey

E. Gülmez, M. Kaya ${ }^{59}$, O. Kaya ${ }^{60}$, S. Tekten, E.A. Yetkin ${ }^{61}$

Istanbul Technical University, Istanbul, Turkey

M.N. Agaras, S. Atay, A. Cakir, K. Cankocak, Y. Komurcu

Institute for Scintillation Materials of National Academy of Science of Ukraine, Kharkov, Ukraine

B. Grynyov

National Scientific Center, Kharkov Institute of Physics and Technology, Kharkov, Ukraine

L. Levchuk

University of Bristol, Bristol, United Kingdom

F. Ball, L. Beck, J.J. Brooke, D. Burns, E. Clement, D. Cussans, O. Davignon, H. Flacher,

J. Goldstein, G.P. Heath, H.F. Heath, L. Kreczko, D.M. Newbold ${ }^{62}$, S. Paramesvaran,

T. Sakuma, S. Seif El Nasr-storey, D. Smith, V.J. Smith 


\section{Rutherford Appleton Laboratory, Didcot, United Kingdom}

K.W. Bell, A. Belyaev ${ }^{63}$, C. Brew, R.M. Brown, D. Cieri, D.J.A. Cockerill, J.A. Coughlan, K. Harder, S. Harper, J. Linacre, E. Olaiya, D. Petyt, C.H. Shepherd-Themistocleous, A. Thea, I.R. Tomalin, T. Williams, W.J. Womersley

\section{Imperial College, London, United Kingdom}

G. Auzinger, R. Bainbridge, P. Bloch, J. Borg, S. Breeze, O. Buchmuller, A. Bundock, S. Casasso, D. Colling, L. Corpe, P. Dauncey, G. Davies, M. Della Negra, R. Di Maria, A. Elwood, Y. Haddad, G. Hall, G. Iles, T. James, M. Komm, R. Lane, C. Laner, L. Lyons, A.-M. Magnan, S. Malik, L. Mastrolorenzo, T. Matsushita, J. $\mathrm{Nash}^{64}$, A. Nikitenko ${ }^{7}$, V. Palladino, M. Pesaresi, A. Richards, A. Rose, E. Scott, C. Seez, A. Shtipliyski, T. Strebler, S. Summers, A. Tapper, K. Uchida, M. Vazquez Acosta ${ }^{65}$, T. Virdee ${ }^{16}$, N. Wardle, D. Winterbottom, J. Wright, S.C. Zenz

Brunel University, Uxbridge, United Kingdom

J.E. Cole, P.R. Hobson, A. Khan, P. Kyberd, A. Morton, I.D. Reid, L. Teodorescu, S. Zahid Baylor University, Waco, U.S.A.

A. Borzou, K. Call, J. Dittmann, K. Hatakeyama, H. Liu, N. Pastika, C. Smith

Catholic University of America, Washington DC, U.S.A.

R. Bartek, A. Dominguez

The University of Alabama, Tuscaloosa, U.S.A.

A. Buccilli, S.I. Cooper, C. Henderson, P. Rumerio, C. West

Boston University, Boston, U.S.A.

D. Arcaro, A. Avetisyan, T. Bose, D. Gastler, D. Rankin, C. Richardson, J. Rohlf, L. Sulak, D. Zou

\section{Brown University, Providence, U.S.A.}

G. Benelli, D. Cutts, M. Hadley, J. Hakala, U. Heintz, J.M. Hogan ${ }^{66}$, K.H.M. Kwok, E. Laird, G. Landsberg, J. Lee, Z. Mao, M. Narain, J. Pazzini, S. Piperov, S. Sagir, R. Syarif, D. Yu

University of California, Davis, Davis, U.S.A.

R. Band, C. Brainerd, R. Breedon, D. Burns, M. Calderon De La Barca Sanchez, M. Chertok, J. Conway, R. Conway, P.T. Cox, R. Erbacher, C. Flores, G. Funk, W. Ko, R. Lander, C. Mclean, M. Mulhearn, D. Pellett, J. Pilot, S. Shalhout, M. Shi, J. Smith, D. Stolp, D. Taylor, K. Tos, M. Tripathi, Z. Wang, F. Zhang

University of California, Los Angeles, U.S.A.

M. Bachtis, C. Bravo, R. Cousins, A. Dasgupta, A. Florent, J. Hauser, M. Ignatenko, N. Mccoll, S. Regnard, D. Saltzberg, C. Schnaible, V. Valuev

University of California, Riverside, Riverside, U.S.A.

E. Bouvier, K. Burt, R. Clare, J. Ellison, J.W. Gary, S.M.A. Ghiasi Shirazi, G. Hanson, G. Karapostoli, E. Kennedy, F. Lacroix, O.R. Long, M. Olmedo Negrete, M.I. Paneva, W. Si, L. Wang, H. Wei, S. Wimpenny, B. R. Yates 
University of California, San Diego, La Jolla, U.S.A.

J.G. Branson, S. Cittolin, M. Derdzinski, R. Gerosa, D. Gilbert, B. Hashemi, A. Holzner, D. Klein, G. Kole, V. Krutelyov, J. Letts, M. Masciovecchio, D. Olivito, S. Padhi, M. Pieri, M. Sani, V. Sharma, S. Simon, M. Tadel, A. Vartak, S. Wasserbaech ${ }^{67}$, J. Wood, F. Würthwein, A. Yagil, G. Zevi Della Porta

University of California, Santa Barbara - Department of Physics, Santa Barbara, U.S.A.

N. Amin, R. Bhandari, J. Bradmiller-Feld, C. Campagnari, M. Citron, A. Dishaw, V. Dutta, M. Franco Sevilla, L. Gouskos, R. Heller, J. Incandela, A. Ovcharova, H. Qu, J. Richman, D. Stuart, I. Suarez, J. Yoo

California Institute of Technology, Pasadena, U.S.A.

D. Anderson, A. Bornheim, J. Bunn, J.M. Lawhorn, H.B. Newman, T. Q. Nguyen, C. Pena, M. Spiropulu, J.R. Vlimant, R. Wilkinson, S. Xie, Z. Zhang, R.Y. Zhu

Carnegie Mellon University, Pittsburgh, U.S.A.

M.B. Andrews, T. Ferguson, T. Mudholkar, M. Paulini, J. Russ, M. Sun, H. Vogel, I. Vorobiev, M. Weinberg

University of Colorado Boulder, Boulder, U.S.A.

J.P. Cumalat, W.T. Ford, F. Jensen, A. Johnson, M. Krohn, S. Leontsinis, E. MacDonald, T. Mulholland, K. Stenson, K.A. Ulmer, S.R. Wagner

Cornell University, Ithaca, U.S.A.

J. Alexander, J. Chaves, Y. Cheng, J. Chu, A. Datta, K. Mcdermott, N. Mirman, J.R. Patterson, D. Quach, A. Rinkevicius, A. Ryd, L. Skinnari, L. Soffi, S.M. Tan, Z. Tao, J. Thom, J. Tucker, P. Wittich, M. Zientek

Fermi National Accelerator Laboratory, Batavia, U.S.A.

S. Abdullin, M. Albrow, M. Alyari, G. Apollinari, A. Apresyan, A. Apyan, S. Banerjee, L.A.T. Bauerdick, A. Beretvas, J. Berryhill, P.C. Bhat, G. Bolla ${ }^{\dagger}$, K. Burkett, J.N. Butler, A. Canepa, G.B. Cerati, H.W.K. Cheung, F. Chlebana, M. Cremonesi, J. Duarte, V.D. Elvira, J. Freeman, Z. Gecse, E. Gottschalk, L. Gray, D. Green, S. Grünendahl, O. Gutsche, J. Hanlon, R.M. Harris, S. Hasegawa, J. Hirschauer, Z. Hu, B. Jayatilaka, S. Jindariani, M. Johnson, U. Joshi, B. Klima, M.J. Kortelainen, B. Kreis, S. Lammel, D. Lincoln, R. Lipton, M. Liu, T. Liu, R. Lopes De Sá, J. Lykken, K. Maeshima, N. Magini, J.M. Marraffino, D. Mason, P. McBride, P. Merkel, S. Mrenna, S. Nahn, V. O'Dell, K. Pedro, O. Prokofyev, G. Rakness, L. Ristori, A. Savoy-Navarro ${ }^{68}$, B. Schneider, E. Sexton-Kennedy, A. Soha, W.J. Spalding, L. Spiegel, S. Stoynev, J. Strait, N. Strobbe, L. Taylor, S. Tkaczyk, N.V. Tran, L. Uplegger, E.W. Vaandering, C. Vernieri, M. Verzocchi, R. Vidal, M. Wang, H.A. Weber, A. Whitbeck, W. Wu

University of Florida, Gainesville, U.S.A.

D. Acosta, P. Avery, P. Bortignon, D. Bourilkov, A. Brinkerhoff, A. Carnes, M. Carver, D. Curry, R.D. Field, I.K. Furic, S.V. Gleyzer, B.M. Joshi, J. Konigsberg, A. Korytov, 
K. Kotov, P. Ma, K. Matchev, H. Mei, G. Mitselmakher, K. Shi, D. Sperka, N. Terentyev, L. Thomas, J. Wang, S. Wang, J. Yelton

Florida International University, Miami, U.S.A.

Y.R. Joshi, S. Linn, P. Markowitz, J.L. Rodriguez

Florida State University, Tallahassee, U.S.A.

A. Ackert, T. Adams, A. Askew, S. Hagopian, V. Hagopian, K.F. Johnson, T. Kolberg, G. Martinez, T. Perry, H. Prosper, A. Saha, A. Santra, V. Sharma, R. Yohay

Florida Institute of Technology, Melbourne, U.S.A.

M.M. Baarmand, V. Bhopatkar, S. Colafranceschi, M. Hohlmann, D. Noonan, T. Roy, F. Yumiceva

University of Illinois at Chicago (UIC), Chicago, U.S.A.

M.R. Adams, L. Apanasevich, D. Berry, R.R. Betts, R. Cavanaugh, X. Chen, S. Dittmer, O. Evdokimov, C.E. Gerber, D.A. Hangal, D.J. Hofman, K. Jung, J. Kamin, I.D. Sandoval Gonzalez, M.B. Tonjes, N. Varelas, H. Wang, Z. Wu, J. Zhang

The University of Iowa, Iowa City, U.S.A.

B. Bilki ${ }^{69}$, W. Clarida, K. Dilsiz ${ }^{70}$, S. Durgut, R.P. Gandrajula, M. Haytmyradov, V. Khristenko, J.-P. Merlo, H. Mermerkaya ${ }^{71}$, A. Mestvirishvili, A. Moeller, J. Nachtman, H. Ogul ${ }^{72}$, Y. Onel, F. Ozok ${ }^{73}$, A. Penzo, C. Snyder, E. Tiras, J. Wetzel, K. Yi

Johns Hopkins University, Baltimore, U.S.A.

B. Blumenfeld, A. Cocoros, N. Eminizer, D. Fehling, L. Feng, A.V. Gritsan, P. Maksimovic, J. Roskes, U. Sarica, M. Swartz, M. Xiao, C. You

The University of Kansas, Lawrence, U.S.A.

A. Al-bataineh, P. Baringer, A. Bean, S. Boren, J. Bowen, J. Castle, S. Khalil, A. Kropivnitskaya, D. Majumder, W. Mcbrayer, M. Murray, C. Rogan, C. Royon, S. Sanders, E. Schmitz, J.D. Tapia Takaki, Q. Wang

Kansas State University, Manhattan, U.S.A.

A. Ivanov, K. Kaadze, Y. Maravin, A. Modak, A. Mohammadi, L.K. Saini, N. Skhirtladze

Lawrence Livermore National Laboratory, Livermore, U.S.A.

F. Rebassoo, D. Wright

University of Maryland, College Park, U.S.A.

A. Baden, O. Baron, A. Belloni, S.C. Eno, Y. Feng, C. Ferraioli, N.J. Hadley, S. Jabeen, G.Y. Jeng, R.G. Kellogg, J. Kunkle, A.C. Mignerey, F. Ricci-Tam, Y.H. Shin, A. Skuja, S.C. Tonwar

\section{Massachusetts Institute of Technology, Cambridge, U.S.A.}

D. Abercrombie, B. Allen, V. Azzolini, R. Barbieri, A. Baty, G. Bauer, R. Bi, S. Brandt, W. Busza, I.A. Cali, M. D’Alfonso, Z. Demiragli, G. Gomez Ceballos, M. Goncharov, P. Harris, D. Hsu, M. Hu, Y. Iiyama, G.M. Innocenti, M. Klute, D. Kovalskyi, Y.-J. Lee, A. Levin, P.D. Luckey, B. Maier, A.C. Marini, C. Mcginn, C. Mironov, S. Narayanan, 
X. Niu, C. Paus, C. Roland, G. Roland, G.S.F. Stephans, K. Sumorok, K. Tatar, D. Velicanu, J. Wang, T.W. Wang, B. Wyslouch, S. Zhaozhong

University of Minnesota, Minneapolis, U.S.A.

A.C. Benvenuti, R.M. Chatterjee, A. Evans, P. Hansen, S. Kalafut, Y. Kubota, Z. Lesko, J. Mans, S. Nourbakhsh, N. Ruckstuhl, R. Rusack, J. Turkewitz, M.A. Wadud

University of Mississippi, Oxford, U.S.A.

J.G. Acosta, S. Oliveros

University of Nebraska-Lincoln, Lincoln, U.S.A.

E. Avdeeva, K. Bloom, D.R. Claes, C. Fangmeier, F. Golf, R. Gonzalez Suarez, R. Kamalieddin, I. Kravchenko, J. Monroy, J.E. Siado, G.R. Snow, B. Stieger

State University of New York at Buffalo, Buffalo, U.S.A.

J. Dolen, A. Godshalk, C. Harrington, I. Iashvili, D. Nguyen, A. Parker, S. Rappoccio, B. Roozbahani

Northeastern University, Boston, U.S.A.

G. Alverson, E. Barberis, C. Freer, A. Hortiangtham, A. Massironi, D.M. Morse, T. Orimoto, R. Teixeira De Lima, T. Wamorkar, B. Wang, A. Wisecarver, D. Wood

Northwestern University, Evanston, U.S.A.

S. Bhattacharya, O. Charaf, K.A. Hahn, N. Mucia, N. Odell, M.H. Schmitt, K. Sung, M. Trovato, M. Velasco

University of Notre Dame, Notre Dame, U.S.A.

R. Bucci, N. Dev, M. Hildreth, K. Hurtado Anampa, C. Jessop, D.J. Karmgard, N. Kellams, K. Lannon, W. Li, N. Loukas, N. Marinelli, F. Meng, C. Mueller, Y. Musienko ${ }^{37}$, M. Planer, A. Reinsvold, R. Ruchti, P. Siddireddy, G. Smith, S. Taroni, M. Wayne, A. Wightman, M. Wolf, A. Woodard

The Ohio State University, Columbus, U.S.A.

J. Alimena, L. Antonelli, B. Bylsma, L.S. Durkin, S. Flowers, B. Francis, A. Hart, C. Hill, W. Ji, T.Y. Ling, W. Luo, B.L. Winer, H.W. Wulsin

Princeton University, Princeton, U.S.A.

S. Cooperstein, O. Driga, P. Elmer, J. Hardenbrook, P. Hebda, S. Higginbotham, A. Kalogeropoulos, D. Lange, J. Luo, D. Marlow, K. Mei, I. Ojalvo, J. Olsen, C. Palmer, P. Piroué, J. Salfeld-Nebgen, D. Stickland, C. Tully

University of Puerto Rico, Mayaguez, U.S.A.

S. Malik, S. Norberg

Purdue University, West Lafayette, U.S.A.

A. Barker, V.E. Barnes, S. Das, L. Gutay, M. Jones, A.W. Jung, A. Khatiwada, D.H. Miller, N. Neumeister, C.C. Peng, H. Qiu, J.F. Schulte, J. Sun, F. Wang, R. Xiao, W. Xie

Purdue University Northwest, Hammond, U.S.A.

T. Cheng, N. Parashar 
Rice University, Houston, U.S.A.

Z. Chen, K.M. Ecklund, S. Freed, F.J.M. Geurts, M. Guilbaud, M. Kilpatrick, W. Li, B. Michlin, B.P. Padley, J. Roberts, J. Rorie, W. Shi, Z. Tu, J. Zabel, A. Zhang

University of Rochester, Rochester, U.S.A.

A. Bodek, P. de Barbaro, R. Demina, Y.t. Duh, T. Ferbel, M. Galanti, A. Garcia-Bellido, J. Han, O. Hindrichs, A. Khukhunaishvili, K.H. Lo, P. Tan, M. Verzetti

The Rockefeller University, New York, U.S.A.

R. Ciesielski, K. Goulianos, C. Mesropian

Rutgers, The State University of New Jersey, Piscataway, U.S.A.

A. Agapitos, J.P. Chou, Y. Gershtein, T.A. Gómez Espinosa, E. Halkiadakis, M. Heindl, E. Hughes, S. Kaplan, R. Kunnawalkam Elayavalli, S. Kyriacou, A. Lath, R. Montalvo, K. Nash, M. Osherson, H. Saka, S. Salur, S. Schnetzer, D. Sheffield, S. Somalwar, R. Stone, S. Thomas, P. Thomassen, M. Walker

University of Tennessee, Knoxville, U.S.A.

A.G. Delannoy, J. Heideman, G. Riley, K. Rose, S. Spanier, K. Thapa

Texas A\&M University, College Station, U.S.A.

O. Bouhali ${ }^{74}$, A. Castaneda Hernandez ${ }^{74}$, A. Celik, M. Dalchenko, M. De Mattia, A. Delgado, S. Dildick, R. Eusebi, J. Gilmore, T. Huang, T. Kamon ${ }^{75}$, R. Mueller, Y. Pakhotin, R. Patel, A. Perloff, L. Perniè, D. Rathjens, A. Safonov, A. Tatarinov

Texas Tech University, Lubbock, U.S.A.

N. Akchurin, J. Damgov, F. De Guio, P.R. Dudero, J. Faulkner, E. Gurpinar, S. Kunori, K. Lamichhane, S.W. Lee, T. Mengke, S. Muthumuni, T. Peltola, S. Undleeb, I. Volobouev, Z. Wang

Vanderbilt University, Nashville, U.S.A.

S. Greene, A. Gurrola, R. Janjam, W. Johns, C. Maguire, A. Melo, H. Ni, K. Padeken, J.D. Ruiz Alvarez, P. Sheldon, S. Tuo, J. Velkovska, Q. Xu

University of Virginia, Charlottesville, U.S.A.

M.W. Arenton, P. Barria, B. Cox, R. Hirosky, M. Joyce, A. Ledovskoy, H. Li, C. Neu, T. Sinthuprasith, Y. Wang, E. Wolfe, F. Xia

Wayne State University, Detroit, U.S.A.

R. Harr, P.E. Karchin, N. Poudyal, J. Sturdy, P. Thapa, S. Zaleski

University of Wisconsin - Madison, Madison, WI, U.S.A.

M. Brodski, J. Buchanan, C. Caillol, D. Carlsmith, S. Dasu, L. Dodd, S. Duric, B. Gomber, M. Grothe, M. Herndon, A. Hervé, U. Hussain, P. Klabbers, A. Lanaro, A. Levine, K. Long, R. Loveless, V. Rekovic, T. Ruggles, A. Savin, N. Smith, W.H. Smith, N. Woods

$\dagger$ : Deceased

1: Also at Vienna University of Technology, Vienna, Austria 
2: Also at IRFU, CEA, Université Paris-Saclay, Gif-sur-Yvette, France

3: Also at Universidade Estadual de Campinas, Campinas, Brazil

4: Also at Federal University of Rio Grande do Sul, Porto Alegre, Brazil

5: Also at Universidade Federal de Pelotas, Pelotas, Brazil

6: Also at Université Libre de Bruxelles, Bruxelles, Belgium

7: Also at Institute for Theoretical and Experimental Physics, Moscow, Russia

8: Also at Joint Institute for Nuclear Research, Dubna, Russia

9: Also at Suez University, Suez, Egypt

10: Now at British University in Egypt, Cairo, Egypt

11: Now at Cairo University, Cairo, Egypt

12: Also at Department of Physics, King Abdulaziz University, Jeddah, Saudi Arabia

13: Also at Université de Haute Alsace, Mulhouse, France

14: Also at Skobeltsyn Institute of Nuclear Physics, Lomonosov Moscow State University, Moscow, Russia

15: Also at Tbilisi State University, Tbilisi, Georgia

16: Also at CERN, European Organization for Nuclear Research, Geneva, Switzerland

17: Also at RWTH Aachen University, III. Physikalisches Institut A, Aachen, Germany

18: Also at University of Hamburg, Hamburg, Germany

19: Also at Brandenburg University of Technology, Cottbus, Germany

20: Also at MTA-ELTE Lendület CMS Particle and Nuclear Physics Group, Eötvös Loránd University, Budapest, Hungary

21: Also at Institute of Nuclear Research ATOMKI, Debrecen, Hungary

22: Also at Institute of Physics, University of Debrecen, Debrecen, Hungary

23: Also at Indian Institute of Technology Bhubaneswar, Bhubaneswar, India

24: Also at Institute of Physics, Bhubaneswar, India

25: Also at Shoolini University, Solan, India

26: Also at University of Visva-Bharati, Santiniketan, India

27: Also at University of Ruhuna, Matara, Sri Lanka

28: Also at Isfahan University of Technology, Isfahan, Iran

29: Also at Yazd University, Yazd, Iran

30: Also at Plasma Physics Research Center, Science and Research Branch, Islamic Azad University, Tehran, Iran

31: Also at Università degli Studi di Siena, Siena, Italy

32: Also at INFN Sezione di Milano-Bicocca; Università di Milano-Bicocca, Milano, Italy

33: Also at International Islamic University of Malaysia, Kuala Lumpur, Malaysia

34: Also at Malaysian Nuclear Agency, MOSTI, Kajang, Malaysia

35: Also at Consejo Nacional de Ciencia y Tecnología, Mexico city, Mexico

36: Also at Warsaw University of Technology, Institute of Electronic Systems, Warsaw, Poland

37: Also at Institute for Nuclear Research, Moscow, Russia

38: Now at National Research Nuclear University 'Moscow Engineering Physics Institute' (MEPhI), Moscow, Russia

39: Also at St. Petersburg State Polytechnical University, St. Petersburg, Russia

40: Also at University of Florida, Gainesville, U.S.A.

41: Also at P.N. Lebedev Physical Institute, Moscow, Russia

42: Also at California Institute of Technology, Pasadena, U.S.A.

43: Also at Budker Institute of Nuclear Physics, Novosibirsk, Russia

44: Also at Faculty of Physics, University of Belgrade, Belgrade, Serbia

45: Also at INFN Sezione di Pavia; Università di Pavia, Pavia, Italy 
46: Also at University of Belgrade, Faculty of Physics and Vinca Institute of Nuclear Sciences, Belgrade, Serbia

47: Also at Scuola Normale e Sezione dell'INFN, Pisa, Italy

48: Also at National and Kapodistrian University of Athens, Athens, Greece

49: Also at Riga Technical University, Riga, Latvia

50: Also at Universität Zürich, Zurich, Switzerland

51: Also at Stefan Meyer Institute for Subatomic Physics (SMI), Vienna, Austria

52: Also at Gaziosmanpasa University, Tokat, Turkey

53: Also at Istanbul Aydin University, Istanbul, Turkey

54: Also at Mersin University, Mersin, Turkey

55: Also at Piri Reis University, Istanbul, Turkey

56: Also at Adiyaman University, Adiyaman, Turkey

57: Also at Izmir Institute of Technology, Izmir, Turkey

58: Also at Necmettin Erbakan University, Konya, Turkey

59: Also at Marmara University, Istanbul, Turkey

60: Also at Kafkas University, Kars, Turkey

61: Also at Istanbul Bilgi University, Istanbul, Turkey

62: Also at Rutherford Appleton Laboratory, Didcot, United Kingdom

63: Also at School of Physics and Astronomy, University of Southampton, Southampton, United Kingdom

64: Also at Monash University, Faculty of Science, Clayton, Australia

65: Also at Instituto de Astrofísica de Canarias, La Laguna, Spain

66: Also at Bethel University, ST. PAUL, U.S.A.

67: Also at Utah Valley University, Orem, U.S.A.

68: Also at Purdue University, West Lafayette, U.S.A.

69: Also at Beykent University, Istanbul, Turkey

70: Also at Bingol University, Bingol, Turkey

71: Also at Erzincan University, Erzincan, Turkey

72: Also at Sinop University, Sinop, Turkey

73: Also at Mimar Sinan University, Istanbul, Istanbul, Turkey

74: Also at Texas A\&M University at Qatar, Doha, Qatar

75: Also at Kyungpook National University, Daegu, Korea 